\title{
THE SQP METHOD FOR CONTROL CONSTRAINED OPTIMAL CONTROL OF THE BURGERS EQUATION*
}

\author{
Fredi TrÖltzsch ${ }^{1}$ And Stefan Volkwein ${ }^{2}$
}

\begin{abstract}
A Lagrange-Newton-SQP method is analyzed for the optimal control of the Burgers equation. Distributed controls are given, which are restricted by pointwise lower and upper bounds. The convergence of the method is proved in appropriate Banach spaces. This proof is based on a weak second-order sufficient optimality condition and the theory of Newton methods for generalized equations in Banach spaces. For the numerical realization a primal-dual active set strategy is applied. Numerical examples are included.
\end{abstract}

Mathematics Subject Classification. 49J20, 49K20, 65Kxx.

Received October 12, 2000. Revised April 20, 2001.

\section{INTRODUCTION}

This paper is concerned with the numerical analysis of a sequential quadratic programming (SQP) method for optimal control problems governed by the Burgers equation, which is a one-dimensional simple model for convection-diffusion phenomena, such as shock waves, supersonic flow about airfoils, traffic flow, acoustic transmission, etc. Distributed controls are considered, and terminal and distributed observation is included in the objective functional. We extend the analysis done in [21] to bilaterally control constraints. Convergence and rate of convergence results are proved. Let us refer to [20], where the convergence of the augmented LagrangeSQP method was shown for the optimal control of the stationary Burgers equation with unrestricted controls. Including first-order sufficient optimality conditions in the considerations, we are able to essentially weaken the second-order sufficient optimality conditions needed to prove the convergence of the method. These sufficient conditions tighten up the gap to the associated necessary ones. We refer to $[17,18]$, where convergence results for a SQP method were proved for optimal control problems governed by semilinear equations.

SQP methods for the optimal control of partial differential equations have been the subject of many papers. We refer, for instance, to [8,22] for the optimal control of a phase field model, to [11] for a class of semilinear elliptic optimal control problems and to [10] for time-dependent fluid flow.

Following recent developments for ordinary differential equations, we adopt here the relation between the SQP method and a generalized Newton method. This approach makes the whole theory more transparent. We

Keywords and phrases: Burgers' equation, SQP methods, generalized Newton's method, primal-dual methods, active set strategy.

* This research was supported in part by DFG-Forschungsschwerpunkt "Echtzeitoptimierung großer Systeme" and by the Fonds zur Förderung der wissenschaftlichen Forschung under "Spezialforschungsbereich Optimierung und Kontrolle", SFB 03.

1 Technische Universität Berlin, Fakultät II - Mathematik und Naturwissenschaften, Sekretariat MA 4-5, Straße des 17 Juni 136, 10623 Berlin, Germany; e-mail: troeltz@math.tu-berlin.de

2 Karl-Franzens-Universität Graz, Institut für Mathematik, Heinrichstrasse 36, 8010 Graz, Austria;

e-mail: stefan.volkwein@uni-graz.at 
are able to apply known results on the convergence of generalized Newton methods in Banach spaces assuming the so-called strong regularity at the optimal reference point. In this way the convergence analysis is shorter, and we are able to concentrate on specific questions arising from the Burgers equation.

Once the convergence of the Newton method is shown, we have to verify the strong regularity by sufficient conditions and to show that the Newton steps can be performed by solving linear-quadratic control problems. This interplay between the Newton method and the SQP method is a specific feature, which cannot be derived from general results in Banach spaces, since we have to discuss pointwise relations.

To compute each SQP step we have to solve a linear-quadratic optimal control problem. This is done by a primal-dual active set algorithm, which is based on a generalized Moreau-Yosida approximation of the indicator function of the admissible controls. The method was developed due to [3] and was extended in [9]. Let us also mention [12], where the primal-dual active set algorithm was applied to parabolic optimal control problems.

Optimal control problems for the Burgers equations are important models for the analysis and the development of numerical algorithms. These investigations can be useful to deal with more complicated problems such as optimal control of the Navier-Stokes equations.

The paper is organized in the following manner. In Section 2 we introduce the optimal control problem for the Burgers equation and the corresponding SQP method. The generalized Newton method is established in Section 3. The strong stability of the generalized equation is proved in Section 4, while Section 5 is devoted to perform the Newton steps by SQP steps. The primal-dual active set strategy is introduced in Section 6 , and numerical examples are presented in the last section.

\section{Optimal CONTRol PROBlem AND SQP MEthod}

Define $\Omega=(0,1) \subset \mathbb{R}$ and, for given $T>0, Q=(0, T) \times \Omega$ and $\Sigma=(0, T) \times \partial \Omega$. We set $V=H_{0}^{1}(\Omega)$, $H=L^{2}(\Omega)$ and identify the Hilbert space $H$ with its dual $H^{\prime}$. On $H$ we use the common natural inner product $(\cdot, \cdot)_{H}$, and endow the Hilbert space $V$ with the inner product

$$
(\varphi, \psi)_{V}=\left(\varphi^{\prime}, \psi^{\prime}\right)_{H} \text { for } \varphi, \psi \in V .
$$

Recall that $V$ is continuously embedded into $C(\bar{\Omega})$, see [1] for instance. Moreover, by $L^{2}(0, T ; V)$ we denote the space of (equivalence classes) of measurable abstract functions $\varphi:[0, T] \rightarrow V$, which are square integrable, i.e.,

$$
\int_{0}^{T}\|\varphi(t)\|_{V}^{2} \mathrm{~d} t<\infty
$$

When $t$ is fixed, the expression $\varphi(t)$ stands for the function $\varphi(t, \cdot)$ considered as a function in $\Omega$ only.

In this work we make use of the Hilbert spaces

$$
W(0, T)=\left\{\varphi \in L^{2}(0, T ; V): \varphi_{t} \in L^{2}\left(0, T ; V^{\prime}\right)\right\}
$$

and

$$
\mathcal{W}(0, T)=\left\{\varphi \in L^{2}\left(0, T ; H^{2}(\Omega) \cap V\right): \varphi_{t} \in L^{2}(0, T ; H)\right\}
$$

supplied with their common inner products, see [4], for instance. Notice that $L^{2}(0, T ; H) \sim L^{2}(Q)$.

The admissible set $U_{\text {ad }} \subset L^{\infty}(Q)$ for the controls is given by

$$
U_{\mathrm{ad}}=\left\{u \in L^{2}(Q): u_{a}(t, x) \leq u(t, x) \leq u_{b}(t, x) \text { a.e. in } Q\right\},
$$

where $u_{a}$ and $u_{b}$ are given functions of $L^{\infty}(Q)$ satisfying $u_{a} \leq u_{b}$ a.e. in $Q$. 
Let $f \in L^{2}(Q)$ be a fixed forcing term and $y_{0} \in V$ be a given initial condition. For controls $u \in L^{2}(Q)$ the state $y$ is defined by the weak solution of the Burgers equation

$$
\begin{aligned}
y_{t}-\nu y_{x x}+y y_{x} & =f+b u & & \text { in } Q, \\
y & =0 & & \text { on } \Sigma, \\
y(0) & =y_{0} & & \text { in } \Omega .
\end{aligned}
$$

Here, $\nu>0$ denotes a viscosity parameter, and $b \in L^{\infty}(Q)$ is a given function expressing location and intensity of the control input. For instance, $b=\chi_{Q_{\diamond}}$ might be chosen, where $Q_{\circ} \subset Q$ is the set of control activity.

Definition 2.1. A function $y$ is called a weak solution to $(2.1)$ if $y \in W(0, T)$ and

$$
\left\langle y_{t}(t), \varphi\right\rangle_{V^{\prime}, V}+\nu(y(t), \varphi)_{V}+\left(y(t) y_{x}(t), \varphi\right)_{H}=((f+b u)(t), \varphi)_{H} \quad \text { for all } \varphi \in V \text { and a.e. } t \in[0, T]
$$

and

$$
(y(0), \chi)_{H}=\left(y_{0}, \chi\right)_{H} \quad \text { for all } \chi \in H .
$$

Notice that $\Omega \subset \mathbb{R}$ ensures $V$ to be continuously embedded into $C(\bar{\Omega})$, therefore $y(t) y_{x}(t) \in H$ a.e. on $[0, T]$. Moreover, $W(0, T)$ is continuously embedded into $C([0, T], H)$, hence the initial value $y(0)$ in $(2.2 \mathrm{~b})$ is a well defined element of $H$. The next theorem ensures the existence of a unique weak solution to the Burgers equation which is even more regular. The proof follows along the lines of that for the unsteady Navier-Stokes equations, see [15] for instance. The a priori estimate (2.3) is proved in the appendix.

Theorem 2.2. For all $u, f \in L^{2}(Q)$ and all $y_{0} \in H$ there exists a unique weak solution $y \in W(0, T)$ to $(2.2)$ satisfying

$$
\|y\|_{W(0, T)} \leq C\left(1+\|b u\|_{L^{2}(Q)}^{2}\right)
$$

with a constant $C>0$ only depending on $y_{0}, b, f$ and $\nu$. Moreover, if $y_{0} \in V$, then $y \in \mathcal{W}(0, T)$ holds.

\section{Remark 2.3.}

a) Since $\mathcal{W}(0, T)$ is continuously embedded into the space of all continuous functions from $[0, T]$ into $V$, denoted by $C([0, T] ; V)$, see [4] for example, we conclude from Theorem 2.2 that $y \in C(\bar{Q})$ holds for $y_{0} \in V$.

b) Since $b \in L^{\infty}(Q)$ holds, we obtain from (2.3)

$$
\|y\|_{W(0, T)} \leq \hat{C}\left(1+\|u\|_{L^{2}(Q)}^{2}\right)
$$

where $\hat{C}=\max \left(1,\|b\|_{L^{\infty}(Q)}\right) C$.

Throughout the Sections 2-6 we assume that $y_{0} \in V$. From Remark 2.3-a) and the estimate (B.9), which is proved in the Appendix, we directly infer the next corollary.

Corollary 2.4. With $u, f \in L^{2}(Q)$ and $y_{0} \in V$ holding the unique solution to $(2.2)$ belongs to $C(\bar{Q})$ and satisfies the estimate

$$
\|y\|_{\mathcal{W}(0, T)}+\|y\|_{C(\bar{Q})} \leq C\left(\|f\|_{L^{2}(Q)}^{2}+\left\|y_{0}\right\|_{V}^{2}+\|b u\|_{L^{2}(Q)}^{2}\right)
$$

for a constant $C>0$. 
Now we proceed by introducing the cost functional

$$
J(y, u)=\frac{1}{2} \int_{Q} \alpha_{Q}\left|y-z_{Q}\right|^{2}+\beta_{Q}|u|^{2} \mathrm{~d} x \mathrm{~d} t+\frac{1}{2} \int_{\Omega} \alpha_{\Omega}\left|y(T)-z_{\Omega}\right|^{2} \mathrm{~d} x,
$$

where $z_{Q} \in L^{2}(Q)$ and $z_{\Omega} \in V$ are given desired states, and $\alpha_{Q}, \beta_{Q} \in L^{\infty}(Q), \alpha_{\Omega} \in W^{1, \infty}(\Omega)$ are non-negative weights such that $\beta_{Q} \geq \beta$ a.e. in $Q$ for a constant $\beta>0$. We also assume $b \neq 0$ on a subset $Q \circ \subseteq Q$ with non-zero measure. The control problem can be written as

$$
\min J(y, u) \text { s.t. }(y, u) \text { solves }(2.2) \text { and } u \in U_{\text {ad }} .
$$

We refer the reader to [21] where $(\mathrm{P})$ was considered with $U_{\mathrm{ad}}=L^{2}(Q)$. The next theorem guarantees the existence of a solution to $(\mathrm{P})$.

Theorem 2.5. Problem (P) has at least one (globally) optimal solution $\left(y^{*}, u^{*}\right)$.

Proof. Let $(y, u) \in W(0, T) \times U_{\text {ad }}$ satisfy (2.2). Then we infer from (2.3) that $y$ is bounded in $W(0, T)$. Hence, there exists a $\zeta \geq 0$ with

$$
\zeta=\inf \left\{J(y, u):(y, u) \in W(0, T) \times U_{\text {ad }} \text { solves }(2.2)\right\} .
$$

This implies the existence of a minimizing sequence $\left\{\left(y^{n}, u^{n}\right)\right\}_{n \in \mathbb{N}}$ in $W(0, T) \times U_{\text {ad }}$ such that $\zeta=\lim _{n \rightarrow \infty} J\left(y^{n}\right.$, $\left.u^{n}\right)$ and $\left(y^{n}, u^{n}\right)$ satisfies (2.2) for all $n \in \mathbb{N}$. Since $U_{\text {ad }}$ is bounded, we infer that there exists $u^{*} \in L^{2}(Q)$ and a subsequence $\left\{u^{n_{k}}\right\}_{k \in \mathbb{N}}$ in $L^{2}(Q)$ with $u^{n_{k}} \rightarrow u^{*}$ as $k$ tends to infinity. Moreover, we find from (2.3) that $y^{n_{k}} \rightarrow y^{*} \in W(0, T)$, possibly after selecting a subsequence again. It was proved in [21] that the pair $\left(y^{*}, u^{*}\right)$ satisfies (2.2). Moreover, the functional $J$ weakly lower semicontinuous with respect to $(y, u)$. This yields $J\left(y^{*}, u^{*}\right)=\zeta$. Since $U_{\mathrm{ad}}$ is closed and convex in $L^{2}(Q)$, we get $u^{*} \in U_{\mathrm{ad}}$. Hence, $u^{*}$ is optimal.

Remark 2.6. From $y_{0} \in V$ and Corollary 2.4 we infer that $y^{*}$ belongs to $\mathcal{W}(0, T)$.

Throughout the paper we make often use of the next proposition, which is proved in the Appendix.

Proposition 2.7. Let $a_{1}, a_{2} \in C([0, T] ; H), v_{0} \in H$, and $g \in L^{2}(Q)$. Then

$$
\begin{aligned}
v_{t}-\nu v_{x x}+a_{1} v+a_{2} v_{x} & =g \\
v & \text { in } Q, \\
v(0) & =v_{0} \text { in } \Omega,
\end{aligned}
$$

has a unique solution $y \in W(0, T)$ satisfying

$$
\|v\|_{W(0, T)} \leq C\left(\left\|v_{0}\right\|_{H}+\|g\|_{L^{2}(Q)}\right) .
$$

If, in addition, $v_{0} \in V$ holds, it follows that $y \in \mathcal{W}(0, T)$, and the estimate

$$
\|v\|_{\mathcal{W}(0, T)} \leq C\left(\left\|v_{0}\right\|_{V}+\|g\|_{L^{2}(Q)}\right)
$$

holds.

Due to an embedding argument, see Remark 2.3-a), we directly derive the following corollary.

Corollary 2.8. With the hypotheses of Proposition 2.7, the solution to (2.4) satisfies

$$
\|v\|_{C(\bar{Q})} \leq C\left(\left\|v_{0}\right\|_{V}+\|g\|_{L^{2}(Q)}\right)
$$

for a constant $C>0$. 
Problem $(\mathrm{P})$ is a non-convex programming problem so that different local minima might occur. Numerical methods will deliver a local minimum close to their starting point. Therefore, we do not restrict our investigations to global solutions of $(\mathrm{P})$. We will assume that a fixed reference solution $\left(y^{*}, u^{*}\right)$ is given satisfying certain first- and second-order optimality conditions (ensuring local optimality of the solution).

The Lagrange functional $\mathcal{L}: W(0, T) \times L^{2}(Q) \times W(0, T) \rightarrow \mathbb{R}$ associated with $(\mathrm{P})$ is defined by

$$
\begin{aligned}
\mathcal{L}(y, u, \lambda)= & J(y, u)-\int_{0}^{T}\left\langle y_{t}(t), \lambda(t)\right\rangle_{V^{\prime}, V}+\nu(y(t), \lambda(t))_{V}+\left(y(t) y_{x}(t), \lambda(t)\right)_{H} \mathrm{~d} t \\
& +\int_{0}^{T}((f+b u)(t), \lambda(t))_{H} \mathrm{~d} t .
\end{aligned}
$$

For each fixed $\lambda \in W(0, T)$, the Lagrangian is twice continuously Fréchet-differentiable with respect to $(y, u) \in$ $W(0, T) \times L^{2}(Q)$ and its second derivative is Lipschitz continuous. Notice that, for fixed $t, y(t) \in V \subset C(\bar{\Omega})$ and $y(t) y_{x}(t) \in H$, hence the inner product $\left(y(t) y_{x}(t), \lambda(t)\right)_{H}$ is defined almost everywhere in $[0, T]$. Moreover, it is integrable on $[0, T]$, since $y \in L^{2}(0, T ; V), y_{x} \in L^{2}(Q)$, and $\lambda \in C([0, T], H)$.

Now we present the first-order necessary optimality conditions for a local solution $\left(y^{*}, u^{*}\right)$ of $(\mathrm{P})$. The pair $\left(y^{*}, u^{*}\right)$ has to satisfy together with an adjoint variable $\lambda^{*} \in W(0, T)$ the state system $(2.1)$, the constraints $u^{*} \in U_{\text {ad }}$, the adjoint system

$$
\begin{aligned}
-\lambda_{t}^{*}-\nu \lambda_{x x}^{*}-y^{*} \lambda_{x}^{*} & =\alpha_{Q}\left(y^{*}-z_{Q}\right) & & \text { in } Q, \\
\lambda^{*} & =0 & & \text { on } \Sigma, \\
\lambda^{*}(T) & =\alpha_{\Omega}\left(y^{*}(T)-z_{\Omega}\right) & & \text { in } \Omega
\end{aligned}
$$

and the variational inequality

$$
\int_{Q}\left(\beta_{Q} u^{*}+b \lambda^{*}\right)\left(u-u^{*}\right) \mathrm{d} x \mathrm{~d} t \geq 0 \text { for all } u \in U_{\text {ad }} .
$$

In the following we shall denote by (OS) the first-order necessary optimality system.

Remark 2.9. Recall that (2.6) is equivalent with

$$
u^{*}(t, x)=P_{[\underline{u}(t, x), \bar{u}(t, x)]}\left(\frac{-b(t, x)}{\beta_{Q}(t, x)} \lambda^{*}(t, x)\right),
$$

where $P_{[\gamma, \delta]}: \mathbb{R} \rightarrow[\gamma, \delta]$ denotes the projection onto the interval $[\gamma, \delta]$.

Proposition 2.10. There exists a unique Lagrange multiplier $\lambda^{*}$ associated with the optimal pair $\left(y^{*}, u^{*}\right)$. Moreover, $\lambda^{*} \in \mathcal{W}(0, T)$ holds and

$$
\left\|\lambda^{*}\right\|_{L^{2}(0, T ; V)} \leq C\left(\left\|\alpha_{\Omega}\left(y^{*}(T)-z_{\Omega}\right)\right\|_{H}+\left\|\alpha_{Q}\left(y^{*}-z_{Q}\right)\right\|_{L^{2}(Q)}\right),
$$

where the constant $C>0$ depends on $\nu, T$ and $y^{*}$.

Proof. Let $\tau=T-t$ and $\tilde{\lambda}^{*}(\tau)=\lambda^{*}(t)$ for $\tau \in[0, T]$. Then problem (2.5) can be transformed into the forward differential equation

$$
\tilde{\lambda}_{\tau}^{*}-\nu \tilde{\lambda}_{x x}^{*}-y^{*}(T-\tau) \tilde{\lambda}_{x}^{*}=\alpha_{Q}(T-\tau)\left(y^{*}(T-\tau)-z_{Q}(T-\tau)\right) \text { in } Q
$$

with the initial condition

$$
\tilde{\lambda}^{*}(0)=\alpha_{\Omega}\left(y^{*}(T)-z_{\Omega}\right) \text { in } \Omega \text {. }
$$


Since $\alpha_{\Omega} \in W^{1, \infty}(\Omega), z_{\Omega} \in V$ by assumption and $y^{*}(T) \in V$ by Theorem 2.2 , we infer that $\alpha_{\Omega}\left(y^{*}(T)-z_{\Omega}\right)$ $\in V$. Hence, the existence of a unique Lagrange multiplier $\lambda^{*} \in \mathcal{W}(0, T)$ satisfying (2.7) follows directly from Proposition 2.7.

In the following we assume that a fixed reference pair $\left(y^{*}, u^{*}\right) \in \mathcal{W}(0, T) \times U_{\text {ad }}$ is given satisfying together with $\lambda^{*} \in \mathcal{W}(0, T)$ the first-order necessary optimality conditions. To guarantee that $\left(y^{*}, u^{*}\right)$ is a local solution to $(\mathrm{P})$ we have to assume some kind of second-order sufficient optimality condition. We shall investigate them along with a first-order sufficient optimality condition. Analogously to [6], for arbitrary but fixed $\sigma>0$, we introduce the set

$$
Q_{\sigma}=\left\{(t, x) \in Q:\left|\beta_{Q}(t, x) u^{*}(t, x)+b(t, x) \lambda^{*}(t, x)\right| \geq \sigma\right\}
$$

Lemma 2.11. The control constraint is active on the set $Q_{\sigma}$.

Proof. It is well known that the variational inequality (9) is equivalent with

$$
\left(\beta_{Q}(t, x) u^{*}(t, x)+b(t, x) \lambda^{*}(t, x)\right)\left(u-u^{*}(t, x)\right) \geq 0 \text { a.e. on } Q
$$

for all real numbers $u$ satisfying $u_{a}(t, x) \leq u \leq u_{b}(t, x)$. From this we obtain

$$
u^{*}(t, x)=\left\{\begin{array}{l}
u_{a} \text { if }\left(\beta_{Q} u^{*}+b \lambda^{*}\right)(t, x)>0 \\
u_{b} \text { if }\left(\beta_{Q} u^{*}+b \lambda^{*}\right)(t, x)<0
\end{array}\right.
$$

Remark 2.12. On $Q_{\sigma}$ the control constraints are strongly active enough. Here we do not need the coercivity of $\mathcal{L}^{\prime \prime}\left(y^{*}, u^{*}, \lambda^{*}\right)$, since the first-order sufficiency ensures local optimality.

The second Fréchet-derivative $\mathcal{L}^{\prime \prime}$ of the Lagrangian with respect to the variable $x=(y, u)$ in directions $h_{i}=\left(y_{i}, u_{i}\right) \in W(0, T) \times L^{2}(Q), i=1,2$, is given by

$$
\mathcal{L}^{\prime \prime}(y, u, \lambda)\left(h_{1}, h_{2}\right)=\int_{\Omega} \alpha_{\Omega} y_{1}(T) y_{2}(T) \mathrm{d} x+\int_{Q} \alpha_{Q} y_{1} y_{2}+\beta_{Q} u_{1} u_{2}-\lambda_{x} y_{1} y_{2} \mathrm{~d} x \mathrm{~d} t
$$

Notice that $\mathcal{L}^{\prime \prime}(y, u, \lambda)$ is a continuous bilinear form. In fact, we estimate

$$
\begin{aligned}
\left|\mathcal{L}^{\prime \prime}(y, u, \lambda)\left(h_{1}, h_{2}\right)\right| \leq & \left\|\alpha_{\Omega}\right\|_{L^{\infty}(\Omega)}\left\|y_{1}(T)\right\|_{H}\left\|y_{2}(T)\right\|_{H}+\left\|\alpha_{Q}\right\|_{L^{\infty}(Q)}\left\|y_{1}\right\|_{L^{2}(Q)}\left\|y_{2}\right\|_{L^{2}(Q)} \\
& +\left\|\beta_{Q}\right\|_{L^{\infty}(Q)}\left\|u_{1}\right\|_{L^{2}(Q)}\left\|u_{2}\right\|_{L^{2}(Q)}+\|\lambda\|_{L^{2}(0, T ; V)}\left\|y_{1}\right\|_{C([0, T] ; H)}\left\|y_{2}\right\|_{L^{2}\left(0, T ; L^{\infty}(\Omega)\right)}
\end{aligned}
$$

Due to embedding arguments there exists a constant depending on $\alpha_{\Omega}, \alpha_{Q}$ and $\lambda$ such that

$$
\left|\mathcal{L}^{\prime \prime}(y, u, \lambda)\left(h_{1}, h_{2}\right)\right| \leq C\left\|h_{1}\right\|_{W(0, T) \times L^{2}(Q)}\left\|h_{2}\right\|_{W(0, T) \times L^{2}(Q)} .
$$

The continuity of $\mathcal{L}^{\prime \prime}$ is essential to prove that second order conditions are sufficient for local optimality. The space of the Lagrange multiplier $\lambda$ must comply with this requirement. In our case, $\lambda \in L^{2}(0, T ; V)$ is needed. We have $\lambda \in W(0, T)$ by the adjoint equation, hence $\lambda$ is sufficiently regular. The decisive estimate is given in the last line before (2.9). Later, in the generalized equation, we rely on the higher regularity $\lambda \in \mathcal{W}(0, T)$. Although a slightly weaker regularity would suffice to deal with this equation, we work in $\mathcal{W}(0, T)$ for convenience.

We make use of the following second-order sufficient optimality condition.

Assumption 1. There are constants $\kappa>0$ and $\sigma>0$ such that

$$
\mathcal{L}^{\prime \prime}\left(y^{*}, u^{*}, \lambda^{*}\right)((y, u),(y, u)) \geq \kappa\|u\|_{L^{2}(Q)}^{2}
$$


for all $(y, u) \in W(0, T) \times L^{2}(Q)$, where $u=0$ on $Q_{\sigma}$, and $y$ is the weak solution to the linearized equation

$$
\begin{aligned}
& y_{t}-\nu y_{x x}+\left(y^{*} y\right)_{x}=b u \text { in } Q \text {, } \\
& y=0 \text { on } \Sigma \text {, } \\
& y(0)=0 \quad \text { in } \Omega \text {. }
\end{aligned}
$$

\section{Remark 2.13.}

a) Due to Proposition 2.7, Assumption 1 implies the existence of a constant $\bar{\kappa}>0$ such that

$$
\mathcal{L}^{\prime \prime}\left(y^{*}, u^{*}, \lambda^{*}\right)((y, u),(y, u)) \geq \bar{\kappa}\left(\|y\|_{W(0, T)}^{2}+\|u\|_{L^{2}(Q)}^{2}\right)
$$

b) On $Q_{\sigma}$ the control is active. Thus, the coercivity of the operator $\mathcal{L}^{\prime \prime}\left(y^{*}, u^{*}, \lambda^{*}\right)$ is only assumed on the set $Q \backslash Q_{\sigma}$, which contains the inactive set and part of the active set, where the constraints are only "weakly" active. For the latter ones, $u \geq 0$ if $\bar{u}=u_{a}$ and $u \leq 0$ if $\bar{u}=u_{b}$ might be required to weaken the second order condition. However, we do not consider this issue here.

c) Proposition 2.14 below justifies Assumption 1.

Let us present a sufficient condition for Assumption 1.

Proposition 2.14. If $\lambda_{x}^{*} \leq \alpha_{Q}$ a.e. in $Q$ or if

$$
\left\|\alpha_{Q}\left(y^{*}-z_{Q}\right)\right\|_{L^{2}(Q)}+\left\|\alpha_{\Omega}\left(y^{*}(T)-z_{\Omega}\right)\right\|_{H}
$$

is sufficiently small, then there is a constant $\kappa>0$ such that

$$
\mathcal{L}^{\prime \prime}\left(y^{*}, u^{*}, \lambda^{*}\right)((y, u),(y, u)) \geq \kappa\|u\|_{L^{2}(Q)}^{2}
$$

for all $(y, u) \in W(0, T) \times L^{2}(Q)$ such that $y$ solves $(2.10)$.

Remark 2.15. Notice that this holds independently of $\sigma$ so that Assumption 1 is fulfilled for $Q_{\sigma}=\emptyset$.

Proof of Proposition 2.14. In the proof we shall use a generic constant $C>0$. Let $(y, u) \in W(0, T) \times L^{2}(Q)$ and $y$ solve (2.10). From Proposition 2.7 it follows that $\|y\|_{W(0, T)} \leq C\|u\|_{L^{2}(Q)}$. Thus,

$$
\mathcal{L}^{\prime \prime}\left(y^{*}, u^{*}, \lambda^{*}\right)((y, u),(y, u)) \geq \int_{Q}\left(\alpha_{Q}-\lambda_{x}^{*}\right) y^{2} \mathrm{~d} x \mathrm{~d} t+\frac{\beta}{2}\|u\|_{L^{2}(Q)}^{2}+\frac{\beta}{2 C}\|y\|_{W(0, T)}^{2} .
$$

If $\lambda_{x}^{*} \leq \alpha_{Q}$ a.e. in $Q$, the claim follows directly with $\kappa=\beta / 2$. On the other hand, applying Hölder's inequality and (2.7) we obtain

$$
\begin{aligned}
\int_{Q} \lambda_{x}^{*} y^{2} \mathrm{~d} x \mathrm{~d} t & \leq\left\|\lambda^{*}\right\|_{L^{2}(0, T ; V)}\|y\|_{C([0, T] ; H)}\|y\|_{L^{2}\left(0, T ; L^{\infty}(\Omega)\right)} \\
& \leq C\left(\left\|\alpha_{\Omega}\left(y^{*}(T)-z_{\Omega}\right)\right\|_{H}+\left\|\alpha_{Q}\left(y^{*}-z_{Q}\right)\right\|_{L^{2}(Q)}\right)\|y\|_{W(0, T)}^{2}
\end{aligned}
$$

Now the claim follows if $\left\|\alpha_{\Omega}\left(y^{*}(T)-z_{\Omega}\right)\right\|_{H}+\left\|\alpha_{Q}\left(y^{*}-z_{Q}\right)\right\|_{L^{2}(Q)} \leq \beta /(2 C)$.

To solve the optimal control problem $(\mathrm{P})$ we apply the SQP method. Suppose that we have already computed $\left(y^{n}, u^{n}, \lambda^{n}\right)$ for some $n \geq 0$ with $y^{n}(0)=y_{0}$. Then the next iterate

$$
\left(y^{n+1}, u^{n+1}\right)=\left(y^{n}, u^{n}\right)+(\delta y, \delta u)
$$


is obtained by the solution of the following linear-quadratic optimal control problem

$$
\begin{aligned}
\min J^{n}(\delta y, \delta u)= & J^{\prime}\left(y^{n}, u^{n}\right)(\delta y, \delta u)+\frac{1}{2} \mathcal{L}^{\prime \prime}\left(y^{n}, u^{n}, \lambda^{n}\right)((\delta y, \delta u),(\delta y, \delta u)) \\
= & \int_{\Omega} \alpha_{\Omega}\left(y^{n}(T)-z_{\Omega}\right) \delta y(T) \mathrm{d} x+\int_{Q} \alpha_{Q}\left(y^{n}-z_{Q}\right) \delta y+\beta_{Q} u^{n} \delta u \mathrm{~d} x \mathrm{~d} t \\
& +\frac{1}{2} \int_{\Omega} \alpha_{\Omega} \delta y(T)^{2} \mathrm{~d} x+\frac{1}{2} \int_{Q} \alpha_{Q} \delta y^{2}+\beta_{Q} \delta u^{2}-\lambda_{x}^{n} \delta y^{2} \mathrm{~d} x \mathrm{~d} t
\end{aligned}
$$

subject to

$$
\begin{array}{rlrl}
\delta y_{t}-\nu \delta y_{x x}+\left(y^{n} \delta y\right)_{x}-b \delta u & =-y_{t}^{n}+\nu y_{x x}^{n} & & \\
& -y^{n} y_{x}^{n}+f+b u^{n} & \text { in } Q, \\
y & =0 & & \text { on } \Sigma, \\
\delta y(0) & =0 & & \text { in } \Omega
\end{array}
$$

and to

$$
u^{n}+\delta u \in U_{\mathrm{ad}}
$$

By $\left(\mathrm{QP}_{n}\right)$ we shall denote the optimal control problem (2.11).

In this work we shall prove that, under natural sufficient conditions, the SQP method exhibits local quadratic convergence for a slight modification of our problem, see Remark 5.5. To perform this analysis, we invoke the concept of generalized equations. The known analysis of Newton's method for generalized equations is an elegant and useful tool to discuss the convergence of the SQP method with reasonable effort. Moreover, we shall report on our numerical experience with the SQP method.

\section{Generalized equation and Newton's method}

Now we proceed by transforming the optimality system (OS) into a generalized equation. For that purpose we define the set-valued mapping $N: L^{\infty}(Q) \rightarrow 2^{L^{\infty}(Q)}$ by

$$
N(u)= \begin{cases}\left\{\psi \in L^{\infty}(Q):(\psi, \tilde{u}-u)_{L^{2}(Q)} \leq 0 \text { for all } \tilde{u} \in U_{\mathrm{ad}}\right\} & \text { if } u \in U_{\mathrm{ad}}, \\ \emptyset & \text { otherwise }\end{cases}
$$

Then, the variational inequality (2.6) can be equivalently expressed by

$$
0 \in \beta_{Q} u+b \lambda+N(u) \text {. }
$$

Remark 3.1. The normal cone to the set $U_{\text {ad }} \subset L^{2}(Q)$, is defined by

$$
N_{U_{\text {ad }}}(u)=\left\{\psi \in L^{2}(Q)^{\prime}:\langle\psi, \tilde{u}-u\rangle_{L^{2}(Q)^{\prime}, L^{2}(Q)} \text { for all } \tilde{u} \in U_{\text {ad }}\right\} .
$$

The set $N(u)$ is the intersection of $N_{U_{\text {ad }}}(u)$ with $L^{\infty}(Q)$ (after identification of $L^{2}(Q)^{\prime}$ with $L^{2}(Q)$ ).

As in the SQP method, the initial condition $y(0)=y_{0}$ is kept fixed in the Newton method. The set-valued mapping $u \mapsto N(u)$ from $L^{\infty}(Q)$ to $2^{L^{\infty}(Q)}$ has closed graph. Let us introduce the space

$$
Y=L^{2}(Q) \times V \times L^{2}(Q) \times V \times L^{\infty}(Q)
$$


endowed with the norm

$$
\|\eta\|_{Y}=\left\|e_{Q}\right\|_{L^{2}(Q)}+\left\|e_{\Omega}\right\|_{V}+\left\|\gamma_{Q}\right\|_{L^{2}(Q)}+\left\|\gamma_{\Omega}\right\|_{V}+\left\|\gamma_{u}\right\|_{L^{\infty}(Q)}
$$

for $\eta=\left(e_{Q}, e_{\Omega}, \gamma_{Q}, \gamma_{\Omega}, \gamma_{u}\right)$. Since $y(0)=y_{0}$ is kept fix in the Newton method, we will study permutations of the form $\eta=\left(e_{Q}, 0, \gamma_{Q}, \gamma_{\Omega}, \gamma_{u}\right)$. Let us define the Banach space $X=\mathcal{W}(0, T) \times L^{\infty}(Q) \times \mathcal{W}(0, T)$ supplied with the norm

$$
\|(y, u, \lambda)\|_{X}=\|y\|_{\mathcal{W}(0, T)}+\|u\|_{L^{\infty}(Q)}+\|\lambda\|_{\mathcal{W}(0, T)} .
$$

Now we introduce the set-valued mapping $\mathcal{T}: X \rightarrow 2^{Y}$ by

$$
\mathcal{T}(w)=(\{0\},\{0\},\{0\},\{0\}, N(u)),
$$

and the function $F: X \rightarrow Y$ by $F=\left(F_{1}, \ldots, F_{5}\right)$, where

$$
\begin{aligned}
& F_{1}(y, u, \lambda)=y_{t}-\nu y_{x x}+y y_{x}-f-b u \\
& F_{2}(y, u, \lambda)=y(0)-y_{0} \\
& F_{3}(y, u, \lambda)=-\lambda_{t}-\nu \lambda_{x x}-y_{x} \lambda-\alpha_{Q}\left(y-z_{Q}\right), \\
& F_{4}(y, u, \lambda)=\lambda(T)-\alpha_{\Omega}\left(y(T)-z_{\Omega}\right), \\
& F_{5}(y, u, \lambda)=\beta_{Q} u+b \lambda .
\end{aligned}
$$

The optimality system is equivalent to the generalized equation

$$
0 \in F(w)+\mathcal{T}(w)
$$

where $F$ is of class $C^{1,1}$, and $\mathcal{T}$ has closed graph. Obviously, $w^{*}=\left(y^{*}, u^{*}, \lambda^{*}\right)$ is a solution to (3.1).

To perform the convergence analysis of the SQP method, we apply (theoretically) the generalized Newton method. Suppose that $w^{n}, n \geq 0$, is already computed. The next iterate $w^{n+1}$ is given by the solution to

$$
0 \in F\left(w^{n}\right)+F^{\prime}\left(w^{n}\right)\left(w-w^{n}\right)+\mathcal{T}(w) .
$$

In the following the set $B_{r}(v)$ denotes an open ball of radius $r>0$ centered at the point $v$. To ensure the convergence of the method we make use of the next definition introduced by Robinson in [14].

Definition 3.2. The generalized equation (3.1) is called strongly regular at $w^{*}$ if there exist $r_{1}>0, r_{2}>0$ and $C_{L}>0$ such that for all perturbations $\eta \in B_{r_{1}}\left(0_{Y}\right)$ the linearized equation

$$
\eta \in F\left(w^{*}\right)+F^{\prime}\left(w^{*}\right)\left(w-w^{*}\right)+\mathcal{T}(w)
$$

has a unique solution $w=w(\eta) \in B_{r_{2}}\left(w^{*}\right)$ satisfying the Lipschitz-property

$$
\left\|w\left(\eta_{1}\right)-w\left(\eta_{2}\right)\right\|_{X} \leq C_{L}\left\|\eta_{1}-\eta_{2}\right\|_{Y} \text { for all } \eta_{1}, \eta_{2} \in B_{r_{1}}\left(0_{Y}\right)
$$

The next theorem states sufficient conditions for the well-posedness of the generalized Newton method, and gives a convergence result. For a proof we refer to [2] in the context of SQP methods and to [5] for generalized equations.

Theorem 3.3. Assume that (3.1) is strongly regular at $w^{*}$. Then there are constants $r>0$ and $C>0$ such that for all starting values $w^{0} \in B_{r}\left(w^{*}\right)$ the generalized Newton method generates a unique sequence $\left\{w^{n}\right\}_{n \in \mathbb{N}}$, which remains in $B_{r}\left(w^{*}\right)$ and satisfies the estimate

$$
\left\|w^{n+1}-w^{*}\right\|_{X} \leq C\left\|w^{n}-w^{*}\right\|_{X}^{2} \text { for } n \geq 0 .
$$


Remark 3.4. Estimate (3.4) expresses local quadratic convergence of the method.

\section{Strong REgUlarity}

This section is devoted to prove that Assumption 1 implies strong regularity of (3.1) at $w^{*}$, if a slightly restricted class of admissible controls is substituted for $U_{\text {ad }}$ (see the definition of $\hat{U}_{\text {ad }}$ below). Therefore, we have to investigate the perturbed generalized equation (3.3). This equation can be interpreted as the optimality system of a linear-quadratic control problem. In fact, equation (3.3) is nothing more than the first-order optimality system for the following auxiliary linear-quadratic problem $\left(\mathrm{QP}_{\eta}\right)$ associated with the perturbation $\eta=\left(e_{Q}, e_{\Omega}, \gamma_{Q}, \gamma_{\Omega}, \gamma_{u}\right) \in Y$ :

$$
\begin{aligned}
\min J(y, u ; \eta)= & \int_{\Omega}\left(\alpha_{\Omega}\left(y^{*}(T)-z_{\Omega}\right)+\gamma_{\Omega}\right) y(T) \mathrm{d} x \\
& +\int_{Q}\left(\alpha_{Q}\left(y^{*}-z_{Q}\right)+\gamma_{Q}\right) y+\left(\beta_{Q} u^{*}+\gamma_{u}\right) u \mathrm{~d} x \mathrm{~d} t \\
& +\frac{1}{2} \int_{\Omega} \alpha_{\Omega}\left(y(T)-y^{*}(T)\right)^{2} \mathrm{~d} x \\
& +\frac{1}{2} \int_{Q} \alpha_{Q}\left(y-y^{*}\right)^{2}+\beta_{Q}\left(u-u^{*}\right)^{2}-\lambda_{x}^{*}\left(y-y^{*}\right)^{2} \mathrm{~d} x \mathrm{~d} t
\end{aligned}
$$

subject to

$$
\begin{array}{rlrl}
y_{t}-\nu y_{x x}+\left(y^{*} y\right)_{x}-y^{*} y_{x}^{*}-f-b u & =e_{Q} & & \text { in } Q, \\
y & =0 & & \text { on } \Sigma, \\
y(0) & =y_{0}+e_{\Omega} & \text { in } \Omega
\end{array}
$$

and to

$$
u \in U_{\text {ad }}
$$

Since the problem is possibly non-convex, we cannot prove strong regularity in $U_{\text {ad. }}$ Therefore, we replace (4.2) by

$$
u \in \hat{U}_{\mathrm{ad}}=\left\{v \in U_{\mathrm{ad}}: v=u^{*} \text { on } Q_{\sigma}\right\}
$$

and denote the associated linear-quadratic problem by $\left(\widehat{Q P}_{\eta}\right)$.

Theorem 4.1. With Assumption 1 holding $\left(\widehat{\mathrm{QP}}_{\eta}\right)$ has a unique solution $(\bar{y}, \bar{u})$ for every $\eta \in Y$.

Proof. Let us split $y=\hat{y}+y^{e}$, where the variable part $\hat{y}$ solves

$$
\begin{aligned}
\hat{y}_{t}-\nu \hat{y}_{x x}+\left(y^{*} \hat{y}\right)_{x} & =b u \text { in } Q, \\
\hat{y} & =0 \text { on } \Sigma, \\
\hat{y}(0) & =0 \quad \text { in } \Omega
\end{aligned}
$$

and the fixed part $y^{e}$ is the weak solution to

$$
\begin{aligned}
y_{t}^{e}-\nu y_{x x}^{e}+\left(y^{*} y^{e}\right)_{x} & =e_{Q}+f+y^{*} y_{x}^{*} & & \text { in } Q, \\
y^{e} & =0 & & \text { on } \Sigma, \\
y^{e}(0) & =e_{\Omega} & & \text { in } \Omega .
\end{aligned}
$$


Notice that

$$
\begin{aligned}
\mathcal{L}^{\prime \prime}\left(y^{*}, u^{*}, \lambda^{*}\right)((y, u),(y, u))= & \mathcal{L}^{\prime \prime}\left(y^{*}, u^{*}, \lambda^{*}\right)((\hat{y}, u),(\hat{y}, u))+2 \mathcal{L}^{\prime \prime}\left(y^{*}, u^{*}, \lambda^{*}\right)\left((\hat{y}, u),\left(y^{e}, 0\right)\right) \\
& +\mathcal{L}^{\prime \prime}\left(y^{*}, u^{*}, \lambda^{*}\right)\left(\left(y^{e}, 0\right),\left(y^{e}, 0\right)\right) .
\end{aligned}
$$

Due to Assumption 1 the first term on the right-hand side is coercive. Since the second term is linear in $(\hat{y}, u)$ and $y^{e}$ is fixed, the claim follows.

The Lagrange functional associated with $\left(\mathrm{QP}_{\eta}\right)$ and $\left(\widehat{\mathrm{QP}}_{\eta}\right)$ is given by

$$
\begin{aligned}
\mathcal{L}(y, u, \lambda ; \eta)= & J(y, u ; \eta)-\int_{0}^{T}\left\langle y_{t}(t), \lambda(t)\right\rangle_{V^{\prime}, V}+\nu(y(t), \lambda(t))_{V}+\left(\left(y^{*} y\right)_{x}(t), \lambda(t)\right)_{H} \mathrm{~d} t \\
& +\int_{0}^{T}\left(\left(y^{*} y_{x}^{*}+f+b u+e_{Q}\right)(t), \lambda(t)\right)_{H} \mathrm{~d} t .
\end{aligned}
$$

Due to the first-order necessary optimality conditions for $\left(\widehat{\mathrm{QP}}_{\eta}\right)$, the adjoint state $\lambda$ satisfies the initial boundary value problem

$$
\begin{aligned}
-\lambda_{t}-\nu \lambda_{x x}-y^{*} \lambda_{x} & =\alpha_{Q} y+\gamma_{Q} & & \text { in } Q, \\
\lambda & =0 & & \text { on } \Sigma, \\
\lambda(T) & =\alpha_{\Omega} y(T)+\gamma_{\Omega} & & \text { in } \Omega
\end{aligned}
$$

in the weak sense.

Corollary 4.2. For all $y \in C([0, T] ; V)$ and $\left(\gamma_{\Omega}, \gamma_{Q}\right) \in V \times L^{2}(Q)$ there exists a unique adjoint state $\lambda \in$ $\mathcal{W}(0, T)$ solving (4.5). Moreover, the following estimates hold

$$
\begin{aligned}
& \|\lambda\|_{W(0, T)} \leq C\left(\|y\|_{C([0, T] ; H)}+\left\|\gamma_{\Omega}\right\|_{H}+\left\|\gamma_{Q}\right\|_{L^{2}(Q)}\right) \\
& \|\lambda\|_{\mathcal{W}(0, T)} \leq C\left(\|y\|_{C([0, T] ; V)}+\left\|\gamma_{\Omega}\right\|_{V}+\left\|\gamma_{Q}\right\|_{L^{2}(Q)}\right)
\end{aligned}
$$

for a constant $C>0$.

Proof. If we transform the time by $\tau=T-t, t \in[0, T]$, the existence of a unique adjoint state as well as both estimates follow from Proposition 2.7.

Let us further introduce the following norms in $X$ and $Y$ by

$$
|w|_{X}=\|y\|_{W(0, T)}+\|u\|_{L^{2}(Q)}+\|\lambda\|_{W(0, T)}
$$

for $w=(y, u, \lambda) \in X$ and

$$
|\eta|_{Y}=\left\|e_{Q}\right\|_{L^{2}(Q)}+\left\|e_{\Omega}\right\|_{H}+\left\|\gamma_{Q}\right\|_{L^{2}(Q)}+\left\|\gamma_{\Omega}\right\|_{H}+\left\|\gamma_{u}\right\|_{L^{2}(Q)}
$$

for $\eta=\left(e_{Q}, e_{\Omega}, \gamma_{Q}, \gamma_{\Omega}, \gamma_{u}\right) \in Y$, respectively. Notice the difference in the notation $|\cdot|_{X}$ and $\|\cdot\|_{X}$.

Theorem 4.3 (L $L^{2}$-stability). Suppose that Assumption 1 holds and that for arbitrary $\eta_{1}, \eta_{2} \in Y$ the pairs $\left(y_{i}, u_{i}\right), i=1,2$, are the solutions to $\left(\widehat{\mathrm{QP}}_{\eta}\right)$ with adjoints $\lambda_{i}$. Then there exists a constant $C>0$ independent on $\eta_{1}$ and $\eta_{2}$ such that

$$
\left|\left(y_{1}, u_{1}, \lambda_{1}\right)-\left(y_{2}, u_{2}, \lambda_{2}\right)\right|_{X} \leq C\left|\eta_{1}-\eta_{2}\right|_{Y}
$$


Proof. Due to the first-order necessary optimality conditions we have

$$
\mathcal{L}^{\prime}\left(y_{\eta}, u_{\eta}, \lambda_{\eta} ; \eta\right)\left(\delta y-y_{\eta}, \delta u-u_{\eta}, \delta \lambda-\lambda_{\eta}\right) \geq 0
$$

for all $(\delta y, \delta u, \delta \lambda) \in W(0, T) \times U_{\text {ad }} \times W(0, T)$. Let $\left(y_{i}, u_{i}, \lambda_{i}\right), i=1,2$, solve $\left(\widehat{\mathrm{QP}}_{\eta}\right)$ for $\eta_{1}$ and $\eta_{2}$, respectively. To shorten notation let us introduce

$$
\begin{aligned}
\left(y_{\eta}, u_{\eta}, \lambda_{\eta}\right) & =\left(y_{2}-y_{1}, u_{2}-u_{1}, \lambda_{2}-\lambda_{1}\right) \\
\left(e_{Q}, e_{\Omega}, \gamma_{Q}, \gamma_{\Omega}, \gamma_{u}\right) & =\left(e_{Q}^{1}-e_{Q}^{2}, e_{\Omega}^{1}-e_{\Omega}^{2}, \gamma_{Q}^{1}-\gamma_{Q}^{2}, \gamma_{\Omega}^{1}-\gamma_{\Omega}^{2}, \gamma_{u}^{1}-\gamma_{u}^{2}\right) .
\end{aligned}
$$

Then we infer from (4.8) that

$$
\begin{aligned}
0 \leq & \mathcal{L}^{\prime}\left(y_{1}, u_{1}, \lambda_{1} ; \eta_{1}\right)\left(y_{2}-y_{1}, u_{2}-u_{1}, \lambda_{2}-\lambda_{1}\right) \\
& +\mathcal{L}^{\prime}\left(y_{2}, u_{2}, \lambda_{2} ; \eta_{2}\right)\left(y_{1}-y_{2}, u_{1}-u_{2}, \lambda_{1}-\lambda_{2}\right) \\
= & \left(\gamma_{\Omega}, y_{\eta}(T)\right)_{H}+\left(\gamma_{Q}, y_{\eta}\right)_{L^{2}(Q)}+\left(\gamma_{u}, u_{\eta}\right)_{L^{2}(Q)} \\
& -\mathcal{L}^{\prime \prime}\left(y^{*}, u^{*}, \lambda^{*}\right)\left(\left(y_{\eta}, u_{\eta}\right),\left(y_{\eta}, u_{\eta}\right)\right) \\
& -\int_{0}^{T}\left\langle y_{\eta}(t), \lambda_{\eta}(t)\right\rangle_{V^{\prime}, V}+\nu\left(y_{\eta}(t), \lambda_{\eta}(t)\right)_{V} \mathrm{~d} t \\
& +\int_{0}^{T}\left(\left(y^{*} y_{\eta}\right)_{x}(t), \lambda_{\eta}(t)\right)_{H}+\left(\left(b u_{\eta}+e_{Q}\right)(t), \lambda_{\eta}(t)\right)_{H} \mathrm{~d} t \\
= & \left(\gamma_{\Omega}, y_{\eta}(T)\right)_{H}+\left(\gamma_{Q}, y_{\eta}\right)_{L^{2}(Q)}+\left(\gamma_{u}, u_{\eta}\right)_{L^{2}(Q)} \\
& -\mathcal{L}^{\prime \prime}\left(y^{*}, u^{*}, \lambda^{*}\right)\left(\left(y_{\eta}, u_{\eta}\right),\left(y_{\eta}, u_{\eta}\right)\right)+\int_{0}^{T}\left(e_{Q}(t), \lambda_{\eta}(t)\right)_{H} \mathrm{~d} t .
\end{aligned}
$$

Notice that $y_{\eta}$ is the weak solution of the following parabolic problem:

$$
\begin{aligned}
\left(y_{\eta}\right)_{t}-\nu\left(y_{\eta}\right)_{x x}+\left(y^{*} y_{\eta}\right)_{x} & =b u_{\eta}+e_{Q} & & \text { in } Q, \\
y_{\eta} & =0 & & \text { on } \Sigma, \\
y_{\eta}(0) & =e_{\Omega} & & \text { in } \Omega .
\end{aligned}
$$

To apply Assumption 1 we split $y_{\eta}=\hat{y}+y^{e}$, where $\hat{y}$ solves (4.3) with $u=u_{\eta}$, and $y^{e}$ is the weak solution to

$$
\begin{aligned}
y_{t}^{e}-\nu y_{x x}^{e}+\left(y^{*} y^{e}\right)_{x} & =e_{Q} \text { in } Q, \\
y^{e} & =0 \text { on } \Sigma, \\
y^{e}(0) & =e_{\Omega} \text { in } \Omega .
\end{aligned}
$$

Applying Proposition 2.7 with $a_{1}=y_{x}^{*}$ and $a_{2}=y^{*}$ we derive from (4.3) and (4.10) the estimates

$$
\|\hat{y}\|_{W(0, T)} \leq C\left\|u_{\eta}\right\|_{L^{2}(Q)} \text { and }\left\|y^{e}\right\|_{W(0, T)} \leq C\left(\left\|e_{\Omega}\right\|_{H}+\left\|e_{Q}\right\|_{L^{2}(Q)}\right) .
$$

These bounds imply

$$
\left\|y_{\eta}\right\|_{W(0, T)} \leq C\left(\left\|u_{\eta}\right\|_{L^{2}(Q)}+\left\|e_{\Omega}\right\|_{H}+\left\|e_{Q}\right\|_{L^{2}(Q)}\right) .
$$

Since $u_{1}, u_{2} \in \hat{U}_{\text {ad }}$, we have $u_{\eta}=0$ on $Q_{\sigma}$. By Assumption 1 we obtain

$$
\mathcal{L}^{\prime \prime}\left(y^{*}, u^{*}, \lambda^{*}\right)\left(\left(\hat{y}, u_{\eta}\right),\left(\hat{y}, u_{\eta}\right)\right) \geq \kappa\left\|u_{\eta}\right\|_{L^{2}(Q)}^{2} .
$$


From this estimate and from (2.9) we arrive at

$$
\begin{aligned}
\mathcal{L}^{\prime \prime}\left(y^{*}, u^{*}, \lambda^{*}\right)\left(\left(y_{\eta}, u_{\eta}\right),\left(y_{\eta}, u_{\eta}\right)\right)= & \mathcal{L}^{\prime \prime}\left(y^{*}, u^{*}, \lambda^{*}\right)\left(\left(\hat{y}, u_{\eta}\right),\left(\hat{y}, u_{\eta}\right)\right)+\mathcal{L}^{\prime \prime}\left(y^{*}, u^{*}, \lambda^{*}\right)\left(\left(y^{e}, 0\right),\left(y^{e}, 0\right)\right) \\
& +2 \mathcal{L}^{\prime \prime}\left(y^{*}, u^{*}, \lambda^{*}\right)\left(\left(\hat{y}, u_{\eta}\right),\left(y^{e}, 0\right)\right) \\
\geq & \kappa\left\|u_{\eta}\right\|_{L^{2}(Q)}^{2}-C\left\|y^{e}\right\|_{W(0, T)}\left(\left\|y^{e}\right\|_{W(0, T)}+\|\hat{y}\|_{W(0, T)}+\left\|u_{\eta}\right\|_{L^{2}(Q)}\right)
\end{aligned}
$$

with a constant $C>0$. Thus, it follows from (4.9) that

$$
\begin{aligned}
\kappa\left\|u_{\eta}\right\|_{L^{2}(Q)}^{2} \leq & C\left(\left\|\gamma_{\Omega}\right\|_{H}+\left\|\gamma_{Q}\right\|_{L^{2}(Q)}+\left\|\gamma_{u}\right\|_{L^{2}(Q)}\right)\left\|y_{\eta}\right\|_{W(0, T)} \\
& +\left\|e_{Q}\right\|_{L^{2}(Q)}\left\|\lambda_{\eta}\right\|_{W(0, T)} \\
& +C\left\|y^{e}\right\|_{W(0, T)}\left(\left\|y^{e}\right\|_{W(0, T)}+\|\hat{y}\|_{W(0, T)}+\left\|u_{\eta}\right\|_{L^{2}(Q)}\right) .
\end{aligned}
$$

Using $(4.6,4.11,4.12)$ and Young's inequality we get

$$
\left\|u_{\eta}\right\|_{L^{2}(Q)} \leq C\left|\eta_{1}-\eta_{2}\right|
$$

for a constant $C>0$. From $(4.6,4.12)$ and (4.14) the claim follows.

The $L^{2}$-estimate of the previous theorem holds for perturbations in $L^{2}$. If they belong to $L^{\infty}$, the result can be improved. Let us use the notation introduced in the proof of Theorem 4.3. From Proposition 2.7 we conclude that

$$
\left\|y_{\eta}\right\|_{\mathcal{W}(0, T)} \leq C\left(\left\|u_{\eta}\right\|_{L^{2}(Q)}+\left\|e_{\Omega}\right\|_{V}+\left\|e_{Q}\right\|_{L^{2}(Q)}\right) \leq C\left\|\eta_{1}-\eta_{2}\right\|_{Y}
$$

Applying (4.7) and (4.15) we obtain

$$
\left\|\lambda_{\eta}\right\|_{\mathcal{W}(0, T)} \leq C\left(\left\|\eta_{1}-\eta_{2}\right\|_{Y}+\left\|\gamma_{Q}\right\|_{L^{2}(Q)}+\left\|\gamma_{\Omega}\right\|_{V}\right)
$$

In Remark 2.9 we introduced the projection $P$. For a.e. $(t, x) \in Q$ we obtain

$$
\left|u_{\eta}(t, x)\right| \leq \frac{|b(t, x)|}{\beta}\left(\left|\lambda_{\eta}(t, x)\right|+\left|\gamma_{u}(t, x)\right|\right),
$$

which implies

$$
\left\|u_{\eta}\right\|_{L^{\infty}(Q)} \leq C\left(\left\|\lambda_{\eta}\right\|_{L^{\infty}(Q)}+\left\|\gamma_{u}\right\|_{L^{\infty}(Q)}\right)
$$

for a constant $C>0$. Using (4.7) we arrive at

$$
\left\|\left(y_{\eta}, u_{\eta}, \lambda_{\eta}\right)\right\|_{X} \leq C\left(\left|\eta_{1}-\eta_{2}\right|_{Y}+\left\|y_{\eta}\right\|_{C([0, T] ; V)}+\left\|\gamma_{Q}\right\|_{L^{2}(Q)}+\left\|\gamma_{\Omega}\right\|_{V}+\left\|\gamma_{u}\right\|_{L^{\infty}(Q)}\right)
$$

for a generic constant $C>0$. Since $\mathcal{W}(0, T)$ is continuously embedded into $C([0, T] ; V)$, we infer from $(4.15)$

$$
\left\|\left(y_{\eta}, u_{\eta}, \lambda_{\eta}\right)\right\|_{X} \leq C\left(\left\|\eta_{1}-\eta_{2}\right\|_{Y}+\left\|\gamma_{Q}\right\|_{L^{2}(Q)}+\left\|\gamma_{\Omega}\right\|_{V}+\left\|\gamma_{u}\right\|_{L^{\infty}(Q)}\right) .
$$

Thus, we have proved the next theorem. 
Theorem 4.4 ( $L^{\infty}$-stability). Suppose that Assumption 1 holds. Let $\left(y_{i}, u_{i}, \lambda_{i}\right)$ be the solutions to $\left(\widehat{\mathrm{QP}}_{\eta}\right)$ for arbitrary $\eta_{i} \in Y, i=1,2$. Then there exists a constant $C>0$ independent on $\eta_{1}$ and $\eta_{2}$ such that

$$
\left\|\left(y_{1}, u_{1}, \lambda_{1}\right)-\left(y_{2}, u_{2}, \lambda_{2}\right)\right\|_{X} \leq C\left\|\eta_{1}-\eta_{2}\right\|_{Y} .
$$

Unfortunately, (4.16) holds only for $u \in \hat{U}_{\text {ad }}$. We are not able to prove (4.16) in $U_{\text {ad }}$. In this case, $J_{\eta}$ might be non-convex and $\left(\mathrm{QP}_{\eta}\right)$ may not have a unique solution. We mention that Proposition 2.14 provides a sufficient condition, where we are able to take $\hat{U}_{\text {ad }}=U_{\text {ad }}$. From Theorem 4.4 we obtain the following result.

Theorem 4.5. Suppose that $w^{*}=\left(y^{*}, u^{*}, \lambda^{*}\right)$ solves the first-order necessary optimality conditions and satisfies Assumption 1. Then the generalized equation (3.1) is strongly regular at the point $w^{*}$ provided that the control set $\hat{U}_{\mathrm{ad}}$ is substituted for $U_{\mathrm{ad}}$ in the definition of the operator $\mathcal{T}$.

\section{The LINEAR-QUADRATIC OPTIMAL CONTROL PROBLEM}

In this section we investigate the linear-quadratic optimal control problem which has to be solved in each level of the SQP method. Since we have the strict regularity only on the set $\hat{U}_{\text {ad }}$ we replace (2.11c) by

$$
u^{n}+\delta u \in \hat{U}_{\mathrm{ad}} .
$$

In contrast to $\left(\mathrm{QP}_{n}\right)$ we shall denote the optimal control problem $(2.11 \mathrm{a}, 2.11 \mathrm{~b})$ and $(5.1)$ by $\left(\widehat{\mathrm{QP}}_{n}\right)$.

Let us choose $\left(y^{0}, u^{0}\right) \in \mathcal{W}(0, T) \times \hat{U}_{\text {ad }}$ such that $y^{0}(0)=y_{0}$ holds. The Newton direction $\delta u$ has to satisfy

$$
u_{a}^{n}:=u_{a}-u^{n} \leq \delta u \leq u_{b}-u^{n}=: u_{b}^{n} \text { a.e. in } Q
$$

and on $Q_{\sigma}$ we have $\delta u=0$. To shorten notation, we set

$$
g^{n}=-y_{t}^{n}+\nu y_{x x}^{n}-y^{n} y_{x}^{n}+f+b u^{n} .
$$

Furthermore, we define $h: Q \rightarrow \mathbb{R}$ by

$$
h=-\frac{b}{\beta_{Q}} \text { in } Q .
$$

The first-order necessary optimality conditions for $\left(\widehat{Q P}_{n}\right)$ are given by the state equation $(2.11 \mathrm{~b})$, the control constraint $u^{n}+\delta u \in \hat{U}_{\text {ad }}$ and the adjoint equation for $\delta \lambda$

$$
\begin{array}{rlrl}
-\delta \lambda_{t}-\nu \delta \lambda_{x x}-y^{n} \delta \lambda_{x} & =\alpha_{Q}\left(y^{n}+\delta y-z_{Q}\right) & & \text { in } Q \\
\delta \lambda & =0 & & \text { on } \Sigma, \\
\delta \lambda(T) & =\alpha_{\Omega}\left(y^{n}(T)+\delta y(T)-z_{\Omega}\right) & \text { in } \Omega .
\end{array}
$$

Proposition 5.1. For every $\delta u \in L^{2}(Q)$ and every $n$ the state equation (2.11b) and the adjoint equation (5.4) admit unique solutions $\delta y, \delta \lambda \in \mathcal{W}(0, T)$.

Proof. By assumption we have $y^{0} \in \mathcal{W}(0, T)$. Setting $a_{1}=y_{x}^{0}$ and $a_{2}=y^{0}$ the existence of a unique $\delta y \in \mathcal{W}(0, T)$ follows from Proposition 2.7. As in the proof of Corollary 4.2 we obtain $\delta \lambda \in \mathcal{W}(0, T)$. Since $y^{1}=y^{0}+\delta y$ $\in \mathcal{W}(0, T)$ the claim follows by an induction argument.

Proposition 5.2. The optimal control problem $\left(\widehat{\mathrm{QP}}_{n}\right)$ admits a unique solution, if $\left|\left(y^{n}, u^{n}, \lambda^{n}\right)-\left(y^{*}, u^{*}, \lambda^{*}\right)\right|_{X}$ is sufficiently small. 
Proof. Using Hölder's inequality and Proposition 2.7 we estimate

$$
\begin{aligned}
\left|\left(\mathcal{L}^{\prime \prime}\left(y^{n}, u^{n}, \lambda^{n}\right)-\mathcal{L}^{\prime \prime}\left(y^{*}, u^{*}, \lambda^{*}\right)\right)((y, u)(y, u))\right| & \leq \int_{\Omega}\left|\lambda_{x}^{n}-\lambda_{x}^{*}\right| y^{2} \mathrm{~d} x \mathrm{~d} t \leq\left\|\lambda_{x}^{n}(t)-\lambda_{x}^{*}(t)\right\|_{H}\|y(t)\|_{H}\|y(t)\|_{L^{\infty}(\Omega)} \\
& \leq\|y\|_{C([0, T] ; H)}\|y\|_{L^{2}(0, T ; V)}\left\|\lambda^{n}-\lambda^{*}\right\|_{L^{2}(0, T ; V)} \\
& \leq C\|u\|_{L^{2}(Q)}^{2}\left\|\lambda^{n}-\lambda^{*}\right\|_{L^{2}(0, T ; V)}
\end{aligned}
$$

for a constant $C>0$. Hence, there exist $\varepsilon>0$ and $\tilde{\kappa}>0$ such that

$$
\mathcal{L}^{\prime \prime}\left(y^{n}, u^{n}, \lambda^{n}\right)((y, u),(y, u)) \geq \tilde{\kappa}\|u\|_{L^{2}(Q)}^{2}
$$

for all $\lambda^{n}$ with $\left\|\lambda^{n}-\lambda^{*}\right\|_{L^{2}(0, T ; V)} \leq \varepsilon$ and for all $(y, u) \in W(0, T) \times L^{2}(Q)$, where $u \in U_{\text {ad }}, u=0$ on $Q_{\sigma}$, and $y$ is the weak solution to the linearized equation

$$
\begin{aligned}
y_{t}-\nu y_{x x}+\left(y^{*} y\right)_{x} & =b u \text { in } Q \\
y & =0 \text { on } \Sigma \\
y(0) & =0 \text { in } \Omega .
\end{aligned}
$$

If $\left\|y^{n}-y^{*}\right\|_{W(0, T)}$ is sufficiently close, (5.5) and (5.6) hold with $y^{*}$ replaced by $y^{n}$ in (5.6). This can be proved in a standard way. For instance, we refer to $[18,19]$. Therefore, the optimal control problem is convex with linear constraints. Thus, the claim follows by standard arguments.

Let us discuss the relationship between the Newton and the SQP method. In the following we denote by $\hat{w}^{n}=\left(\hat{y}^{n}, \hat{u}^{n}, \hat{\lambda}^{n}\right)$ the iterates generated by the SQP method performed on $\hat{U}_{\text {ad }}$. The iterates of the generalized Newton method are $w^{n}=\left(y^{n}, u^{n}, \lambda^{n}\right)$. We investigate both methods initiating from the same starting value $w^{n}=\hat{w}^{n}$. If $\left\|w^{n}-w^{*}\right\|_{X}$ is sufficiently small then there exists a unique solution $\left(\hat{y}^{n+1}, \hat{u}^{n+1}\right)$ to $\left(\widehat{\mathrm{QP}}_{n}\right)$ with an associated Lagrange multiplier $\hat{\lambda}^{n+1}$ due to Proposition 5.2. On the other hand, $\hat{w}^{n+1}=\left(\hat{y}^{n+1}, \hat{u}^{n+1}, \hat{\lambda}^{n+1}\right)$ solves the generalized equation (3.2) at $w^{n}$ (based on the set $\hat{U}_{\text {ad }}$ ). For $\left\|w^{n}-w^{*}\right\|_{X}<\hat{r}$, one step of the generalized Newton method delivers the unique solution of (3.2) by Theorem 3.3. As $\hat{w}^{n+1}$ solves the generalized equation, which is locally unique, we get $w^{n+1}=\hat{w}^{n+1}$. If $\left\|w^{n}-w^{*}\right\|_{X}<\min (r, \hat{r})=: \varrho$, then Theorem 3.3 implies that $w^{n+1} \in B_{\varrho}\left(w^{*}\right)$. Thus, $\left\|\hat{w}^{n+1}-w^{*}\right\|_{X}<\varrho$. Hence, we are able to perform the next step in both of the methods. Moreover, Newton's and SQP method are identical on $\hat{U}_{\text {ad }}$.

Theorem 5.3. Let $w^{*}=\left(y^{*}, u^{*}, \lambda^{*}\right)$ satisfy the optimality system (OS) together with Assumption 1. Suppose that a starting value $w^{0}=\left(y^{0}, u^{0}, \lambda^{0}\right) \in X$ is given with $u^{0} \in \hat{U}_{\mathrm{ad}}$ and $\left\|w^{0}-w^{*}\right\|_{X}<\varrho$. Then the generalized Newton method is equivalent to the SQP method in $\hat{U}_{\text {ad }}$.

The next corollary follows directly from Theorems 3.3 and 5.3.

Corollary 5.4. With the assumptions of Theorem 5.3 we have for all $n \in \mathbb{N}$

$$
\left\|\left(y^{n+1}, u^{n+1}, \lambda^{n+1}\right)-\left(y^{*}, u^{*}, \lambda^{*}\right)\right\|_{X} \leq C\left\|\left(y^{n}, u^{n}, \lambda^{n}\right)-\left(y^{*}, u^{*}, \lambda^{*}\right)\right\|_{X}^{2},
$$

where $C>0$ is a constant and $\left(y^{n}, u^{n}, \lambda^{n}\right)$ are the iterates generated by the generalized Newton or SQP method.

Remark 5.5. This convergence result still contains a formal obstacle for the numerical application. It requires the a priori knowledge of a set $Q_{\sigma}$ for some $\sigma>0$, hence the unknown solution $w^{*}$ should be known in advance. Let us briefly explain a way out of this difficulty. Newton's method is known to be only locally convergent. Hence we have to start the iteration in a neighborhood of the unknown solution. In our SQP method, we cannot 
do better, and we must assume additionally that the iterates stay in this neighborhood (see the comments and the counterexample for this issue in [7]). Suppose that the second order sufficient condition is satisfied with $\sigma>0$. Let $w^{0}$ be the initial iterate and define $U_{a d}^{\varepsilon}=\left\{u \in U_{a d} \mid\left\|u-u^{0}\right\|_{L^{\infty}(Q)}<\varepsilon\right\}, \varepsilon$ sufficiently small. If $u^{0}$ is close to $u^{*}$, then all elements $u \in U_{a d}^{\varepsilon}$ are close to $u^{*}$. In this case one can show that $\left(\mathrm{QP}_{n}\right)$, based on $U_{\text {ad }}^{\varepsilon}$ instead on $\hat{U}_{\text {ad }}$, has exactly one solution $u^{n+1}$, which is active on $Q_{\sigma}$ and equal to $u^{*}$ there, provided that $\varepsilon$ is sufficiently small. We do not discuss this intuitively clear observation and refer to the analysis in [18] for a similar parabolic problem. In the numerical application we therefore have two ways to guarantee convergence. We might add an additional constraint of the form $-\varepsilon \leq u-u^{0} \leq \varepsilon$ forcing the algorithm to stay in a neighborhood. Alternatively, we can do without this, if the algorithm is monitored to converge. The latter is what we observed in our numerical tests.

\section{A PRIMAL-DUAL ACTIVE SET ALGORIthm}

To solve the optimal control problems $\left(\mathrm{QP}_{n}\right)$, in each level of the SQP method we use a primal-dual active set strategy. This algorithm is based on a generalized Moreau-Yosida approximation of the indicator function of the set $U_{\text {ad }}$ of admissible controls. For more details we refer to [3].

Let the superscript $n$ and the subscript $k$ denote the current SQP- and active set iteration, respectively, and dual variables $\delta \xi_{k}$ stand for the Lagrange multipliers associated with the inequality constraints

$$
u^{n}+\delta u \in U_{\mathrm{ad}}
$$

Suppose that $\left(\delta u_{k-1}, \delta \xi_{k-1}\right)$ are given. Then the $u_{a}^{n}$-active and $u_{b}^{n}$-active sets of the current iterate are chosen according to

$$
\begin{aligned}
& \underline{A}_{k}^{n}=\left\{(t, x) \in Q: \delta u_{k-1}(t, x)+\frac{\delta \xi_{k-1}(t, x)}{c}<u_{a}^{n}(t, x) \text { a.e. in } \mathrm{Q}\right\} \\
& \bar{A}_{k}^{n}=\left\{(t, x) \in Q: \delta u_{k-1}(t, x)+\frac{\delta \xi_{k-1}(t, x)}{c}>u_{b}^{n}(t, x) \text { a.e. in } \mathrm{Q}\right\}
\end{aligned}
$$

where $c>0$ is a scalar, and we set $A_{k}^{n}=\underline{A}_{k}^{n} \cup \bar{A}_{k}^{n}$. Furthermore, we define the inactive set

$$
I_{k}^{n}=\left\{(t, x) \in Q: u_{a}^{n}(t, x) \leq \delta u_{k-1}(t, x)+\frac{\delta \xi_{k-1}(t, x)}{c} \leq u_{b}^{n}(t, x) \text { a.e. in } \mathrm{Q}\right\}
$$

Notice that, in general, $u^{n}+\delta u_{k-1}$ need not be feasible on $I_{k}^{n}$. Notice that the definition of $A_{k}^{n}$ and $I_{k}^{n}$ involve the primal variable $\delta u$ as well as the dual variable $\delta \xi$ corresponding to the inequality constraints. In Algorithm 1 below the identification $A_{k-1}^{n}=A_{k}^{n}$ means $\underline{A}_{k}^{n}=\underline{A}_{k-1}^{n}$ and $\bar{A}_{k}^{n}=\bar{A}_{k-1}^{n}$.

Algorithm 1 (Primal-dual active set strategy).

a) Choose $c>0$ and starting values $\left(\delta u_{0}, \delta \xi_{0}\right) \in U_{\text {ad }} \times L^{\infty}(Q)$, and set $k=1$;

b) compute $\underline{A}_{k}^{n}, \bar{A}_{k}^{n}$, and $I_{k}^{n}$;

c) if $k \geq 2, A_{k}^{n}=A_{k-1}^{n}, I_{k}^{n}=I_{k-1}^{n}$ then $S T O P$; 
d) else, find $(y, \lambda) \in \mathcal{W}(0, T) \times \mathcal{W}(0, T)$ satisfying

$$
\begin{array}{rlrl}
y_{t}-\nu y_{x x}+\left(y^{n} y\right)_{x} & =b u_{b}^{n}+g^{n} & & \text { in } \bar{A}_{k}^{n}, \\
y_{t}-\nu y_{x x}+\left(y^{n} y\right)_{x} & =b u_{a}^{n}+g^{n} & & \text { in } \underline{A}_{k}^{n}, \\
y_{t}-\nu y_{x x}+\left(y^{n} y\right)_{x}-h \lambda & =g^{n} & & \text { in } I_{k}, \\
y & =0 & & \text { on } \Sigma, \\
y(0) & =0 & & \text { in } \Omega, \\
-\alpha_{Q} y+\lambda_{x} y-\lambda_{t}-\nu \lambda_{x x}-y^{n} \lambda_{x} & =\alpha_{Q}\left(y^{n}-z_{Q}\right) & & \text { in } Q, \\
\lambda & =0 & & \text { on } \Sigma, \\
\alpha_{\Omega} y(T)+\lambda(T) & =\alpha_{\Omega}\left(y^{n}(T)-z_{\Omega}\right) & \text { in } \Omega,
\end{array}
$$

set $\left(\delta y_{k}, \delta \lambda_{k}\right)=(y, \lambda)$ and

$$
\delta u_{k}= \begin{cases}u_{b}^{n} \quad \text { in } \bar{A}_{k}^{n} \\ u_{a}^{n} \quad \text { in } \underline{A}_{k}^{n} \\ h \delta \lambda_{k} & \text { in } I_{k}^{n}\end{cases}
$$

e) put $\delta \xi_{k}=\delta \lambda_{k}-\beta_{Q} \delta u_{k}, k=k+1$, and return to b).

Remark 6.1. Let us mention that Algorithm 1 stops feasible if there exists an iteration level $k$ such that $A_{k}^{n}=A_{k+1}^{n}$. In particular, in this case we have $u^{n}+\delta u_{k} \in U_{\mathrm{ad}}$.

Proposition 6.2. With the hypotheses of Proposition 2.14 there exists a radius $\hat{r}>0$ so that Algorithm 1 is well-defined if

$$
\left\|\left(y^{n}, u^{n}, \lambda^{n}\right)-\left(y^{*}, u^{*}, \lambda^{*}\right)\right\|_{X}<\hat{r} .
$$

Proof. Due to Proposition 2.14 and Remark 2.15 the second-order sufficient optimality condition holds at $\left(y^{*}, u^{*}, \lambda^{*}\right)$ for $Q_{\sigma}=\emptyset$. Using the regularity properties of the Lagrangian, we can prove that there exists a radius $\hat{r}>0$ such that the second-order sufficient optimality condition holds for all $\left(y^{n}, u^{n}, \lambda^{n}\right) \in B_{\hat{r}}\left(y^{*}, u^{*}, \lambda^{*}\right)$, see the proof of Proposition 5.2. Hence, the linear system (6.1) is uniquely solvable.

\section{NumERICAL EXPERIMENTS}

To solve $(\mathrm{P})$ we use the so-called "optimize-then-discretize" approach, i.e., we compute the solution of the linear-quadratic subproblems $\left(\mathrm{QP}_{n}\right)$ by discretizing Algorithm 1, i.e., by discretizing the associated systems (6.1).

In our test runs we also compare the optimal solutions with the solutions of the unconstrained problems, i.e., for $U_{\text {ad }}=L^{2}(Q)$.

For the time integration we apply the backward Euler scheme while the spatial variable is approximated by piecewise linear finite elements. The programs are written in MATLAB, version 5.3, executed on a Pentium III $550 \mathrm{MHz}$ personal computer.

Run 1. In the first test example we choose $T=1, \nu=0.01, y_{0}=0$, and $f=0$. For $N, M \in \mathbb{N}$ the grid was given by

$$
x_{i}=\frac{i}{N} \text { for } i=0, \ldots, N \text { and } t_{j}=\frac{j T}{M} \text { for } j=0, \ldots, M
$$




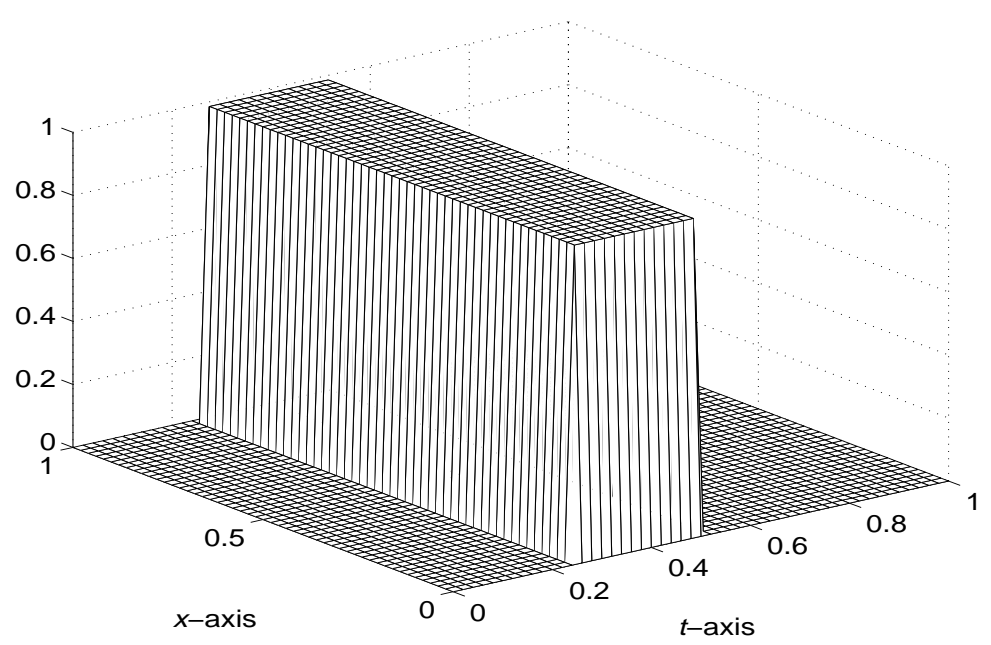

Figure 1. Desired state $z_{Q}$ for $N=M=50$.

Clearly, the solution to (2.2a) with $u=0$ is $y=0$. For the optimal control problem we take $\alpha_{\Omega}=0, \alpha_{Q}=1$, $\beta_{Q}=0.0175$ and $b=\chi_{Q_{\circ}}$, where $\chi_{Q_{\circ}}$ denotes the characteristic function of the set $Q_{\circ}=(0, T / 2) \times(0.5,0.75)$. The desired state is shown in Figure 1.

(i) First we solve the optimal control problem with $U_{\text {ad }}=L^{2}(Q)$ by applying the SQP method. Then the solution $(\delta y, \delta u)$ of $\left(\mathrm{QP}_{n}\right)$ as follows: First we solve the linear system

$$
\begin{array}{rlrl}
(\delta y)_{t}-\nu(\delta y)_{x x}+\left(y^{n} \delta y\right)_{x}-h \delta \lambda & =g^{n} & & \text { in } Q, \\
\delta y & =0 & & \text { on } \Sigma, \\
\delta y(0) & =0 & & \text { in } \Omega, \\
\left(\lambda_{x}^{n}-\alpha_{Q}\right) \delta y-(\delta \lambda)_{t}-\nu(\delta \lambda)_{x x}-y^{n}(\delta \lambda)_{x} & =\alpha_{Q}\left(y^{n}-z_{Q}\right) & & \text { in } Q \\
\delta \lambda & =0 & & \text { on } \Sigma, \\
\alpha_{\Omega} \delta y(T)+\delta \lambda(T) & =\alpha_{\Omega}\left(y^{n}(T)-z_{\Omega}\right) & \text { in } \Omega,
\end{array}
$$

where $g^{n}$ and $h$ were introduced in (5.2) and (5.3), respectively. Next, we obtain $\delta u$ from

$$
\delta u=h \delta \lambda \text { in } Q .
$$

The discretization of (7.1a) leads to an indefinite system $H^{n}(\delta y, \delta \lambda)^{\top}=r^{n}$, where $H^{n}$ is of the form

$$
H^{n}=\left(\begin{array}{cc}
A^{n} & \left(B^{n}\right)^{\top} \\
B^{n} & C^{n}
\end{array}\right)
$$

As starting values we take $y^{0}=0, u^{0}=0$ and $\lambda^{0}=0$. We stop the SQP iteration if the associated residuum is less than $10^{-6}$, i.e.,

$$
\operatorname{Res}(n)=\left\|\nabla \mathcal{L}\left(y^{n}, u^{n}, \lambda^{n}\right)\right\|_{L^{2}(Q)^{3}} \leq 10^{-6}
$$

Here, $\nabla$ stands for the gradient of $\mathcal{L}$ with respect to $(y, u, \lambda)$. Notice that $\nabla_{u} \mathcal{L}\left(y^{n}, u^{n}, \lambda^{n}\right)=0$ is guaranteed by (7.1b). We do not have to check it numerically. 

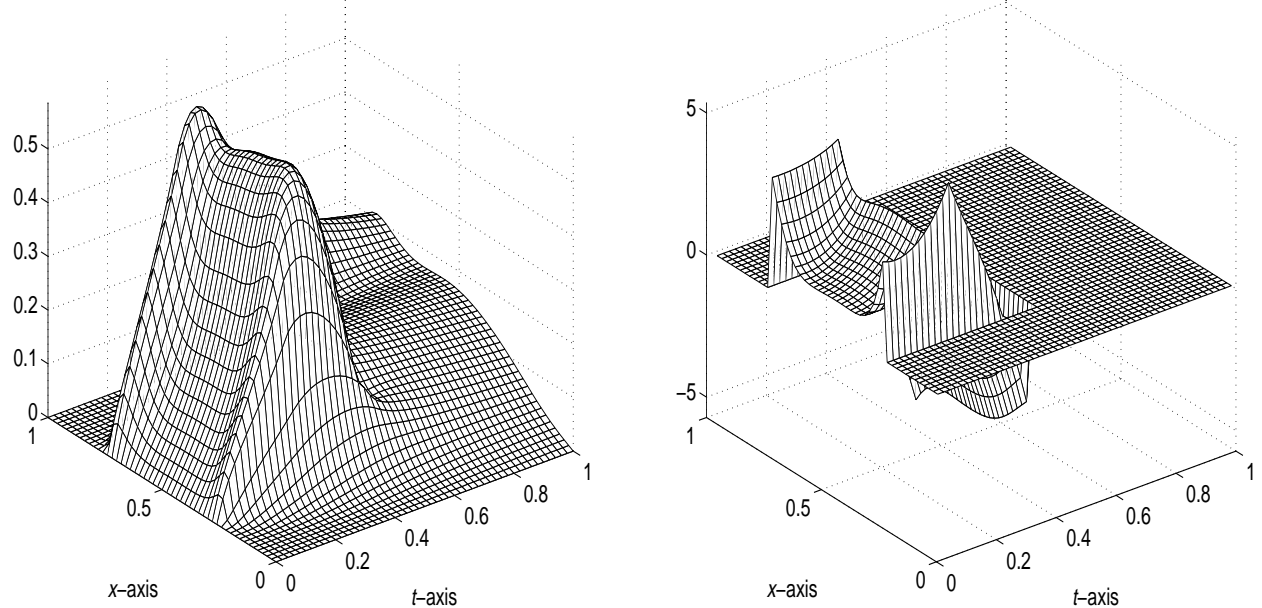

Figure 2. Optimal state and control for $U_{\text {ad }}=L^{2}(Q)$ and $N=M=50$.

TABLE 1. CPU-times in seconds.

\begin{tabular}{|c||c|c|c|}
\hline & (SQP-LU) & (SQP-GMRES) & (SQP-GMRES-IN) \\
\hline$N=M=50$ & 137 & 66 & 59 \\
\hline$N=M=60$ & 249 & 116 & 105 \\
\hline
\end{tabular}

The linear system (7.1a), which has to be solved in each level of the SQP-iteration, is treated in three different ways:

1.) (SQP-LU): utilize a $L U$-factorization with pivoting (MATLAB routine $l u$ ),

2.) (SQP-GMRES): apply the Generalized Minimum Residual Method (MATLAB routine gmres) and stop the iteration if the relative residual

$$
\frac{\left\|r^{n}-H^{n}(\delta y, \delta \lambda)^{\top}\right\|_{2}}{\left\|r^{n}\right\|_{2}}
$$

is less than $10^{-6}$. Here, $\|\cdot\|_{2}$ denotes the Euclidean norm.

3.) (SQP-GMRES-IN): use the GMRES method and stop the iteration if the relative residual is less than the actual value of $\operatorname{Res}(n)$. In this way, the precision for solving the linear system (7.1a) is adapted to the size of the outer iteration. The closer the iterates are to the optimum, the finer the system is solved.

As a preconditioner for the GMRES method we took an incomplete $L U$-factorization of the matrix

$$
D=\left(\begin{array}{cc}
0 & P^{\top} \\
P & 0
\end{array}\right)
$$

by utilizing the MATLAB function luinc(D,1e-05). Here, the matrix $P$ is the discretization of the heat operator $y_{t}-\nu y_{x x}$ with homogeneous Dirichlet boundary conditions at $x=0$ and $x=1$, and the matrix $P^{\top}$ is the discretization of its adjoint. In Figure 2 the discrete optimal solution is presented.

All the three variants of the SQP method stop after five iterations and their optimal solutions coincide. Let us mention that no step size control is necessary in this example. The needed CPU times are given in Table 1. It turns out that for this example the inexact GMRES method is the fastest method. 
TABLE 2. Number of inner iterations, residua, and CPU times for (SQP-LU).

\begin{tabular}{|c||c|r|r|}
\hline SQP iterations & Alg. 1 iterations & residuum & CPU time \\
\hline 1 & 6 & $6.19 \mathrm{e}-03$ & 90.9 seconds \\
\hline 2 & 3 & $6.81 \mathrm{e}-04$ & 76.7 seconds \\
\hline 3 & 2 & $2.23 \mathrm{e}-06$ & 52.2 seconds \\
\hline 4 & 1 & $3.14 \mathrm{e}-09$ & 26.7 seconds \\
\hline \multicolumn{2}{|l}{} & &
\end{tabular}

TABLE 3. Number of inner iterations, residua, and CPU times for (SQP-GMRES).

\begin{tabular}{|c|c|c|c|}
\hline SQP iterations & Alg. 1 iterations & residuum & CPU time \\
\hline 1 & 6 & $6.19 \mathrm{e}-03$ & 63.2 seconds \\
\hline 2 & 3 & $6.81 \mathrm{e}-04$ & 33.4 seconds \\
\hline 3 & 2 & $2.23 \mathrm{e}-06$ & 21.7 seconds \\
\hline 4 & 1 & $3.14 \mathrm{e}-09$ & 11.3 seconds \\
\hline
\end{tabular}

TABLE 4. Number of inner iterations, residua, and CPU times for (SQP-GMRES-IN).

\begin{tabular}{|c|c|c|c|}
\hline SQP iterations & Alg. 1 iterations & residuum & CPU time \\
\hline 1 & 6 & $6.18 \mathrm{e}-03$ & 47.2 seconds \\
\hline 2 & $\overline{4}$ & $6.65 \mathrm{e}-04$ & 30.0 seconds \\
\hline 3 & 2 & $2.08 \mathrm{e}-06$ & 15.8 seconds \\
\hline$\overline{4}$ & 3 & $4.88 \mathrm{e}-09$ & 27.3 seconds \\
\hline
\end{tabular}

TABLE 5. CPU times in seconds.

\begin{tabular}{|c||c|c|c|}
\hline & (SQP-LU) & (SQP-GMRES) & (SQP-GMRES-IN) \\
\hline$N=M=60$ & 673 & 355 & 272 \\
\hline
\end{tabular}

(ii) Next we introduce inequality constraints by choosing $u_{a}=0$ and $u_{b}=1$. We solve the linear system (6.1) utilizing analogous versions of (SQP-LU), (SQP-GMRES) and (SQP-GMRES-IN). For $N=M=50$ the behavior of the SQP-method combined with Algorithm 1 is presented in Tables 2-4. We check Res $(n)$ $\leq 10^{-6}$ only on the current inactive set for $u$. By our active set strategy, the variational inequality is automatically satisfied on the remaining set. Therefore, the necessary optimality conditions are satisfied up to precision $10^{-6}$.

The needed CPU times are given in Table 5 for $N=M=60$. Again, for a fine grid the inexact GMRES method SQP-GMRES-IN is the fastest variant.

The discrete optimal solution is plotted in Figure 3. It turns out that (6.1) is uniquely solvable throughout the iteration process. Therefore, Algorithm 1 is well-defined for $Q_{\sigma}=\emptyset$. The values of the cost functional are presented in Table 6 .

Run 2. In the second test we want to track the optimal state to a desired state at the terminal time. We take $T=1, \nu=0.1$,

$$
y_{0}=\left\{\begin{array}{l}
1 \text { in }(0,0.5] \\
0 \text { otherwise }
\end{array}\right.
$$



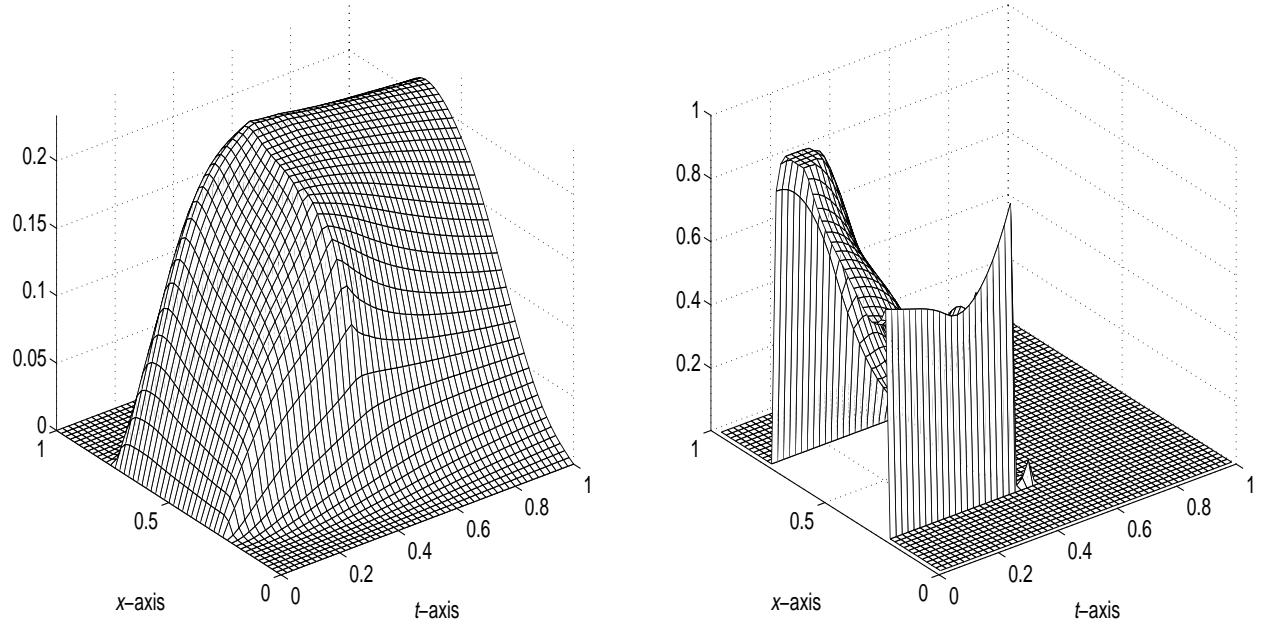

Figure 3. Optimal state and control for $u_{a}=0, u_{b}=1$, and $N=M=50$.

TABLE 6. Values of the cost functional for $N=M=50$.

\begin{tabular}{|c||c|c|c|}
\hline & $u=0$ & $u \in L^{2}(Q)$ & $u \in U_{\text {ad }}$ \\
\hline$J\left(y^{*}, u^{*}\right)$ & 0.12653 & 0.09319 & 0.10743 \\
\hline
\end{tabular}

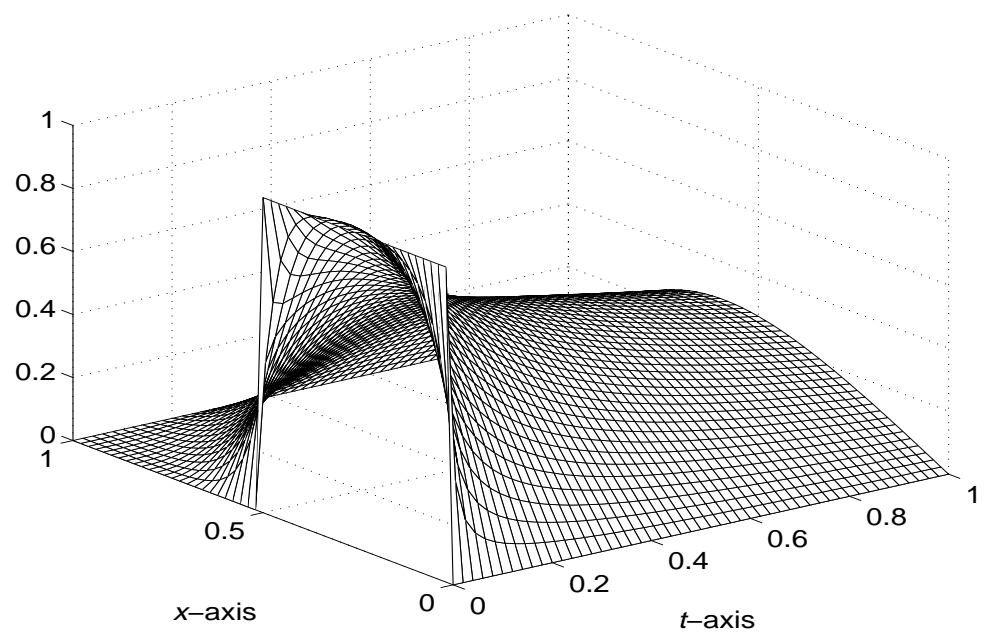

Figure 4. Solution for $u=0$ and $N=M=50$.

and $f=0$. The grid is the same as in the previous test example. To solve (2.1) with $u=0$ we apply Newton's method at each time step. The algorithm needs 1 second in case of $N=M=50$. The numerical solution is shown in Figure 4.

Now we turn to the optimal control problem. We choose $\alpha_{\Omega}=1, \alpha_{Q}=0, \beta_{Q}=0.2$ and $b=\chi_{Q}$ 。 with $Q_{\circ}=(0,9 T / 10) \times(0,0.75)$. The desired state is $z_{\Omega}(x)=\sin (2 \pi x)$. First we solve $(\mathrm{P})$ with $U_{\text {ad }}=L^{2}(Q)$. The starting values for the SQP method are $y^{0}=0, u^{0}=0$ and $\lambda^{0}=0$. The stopping criterion is the same as 
TABLE 7. CPU-times in seconds.

\begin{tabular}{|l||c|c|c|}
\hline & (SQP-LU) & (SQP-GMRES) & (SQP-GMRES-IN) \\
\hline$N=M=50$ & 128 & 58 & 53 \\
\hline$N=M=60$ & 299 & 126 & 118 \\
\hline
\end{tabular}
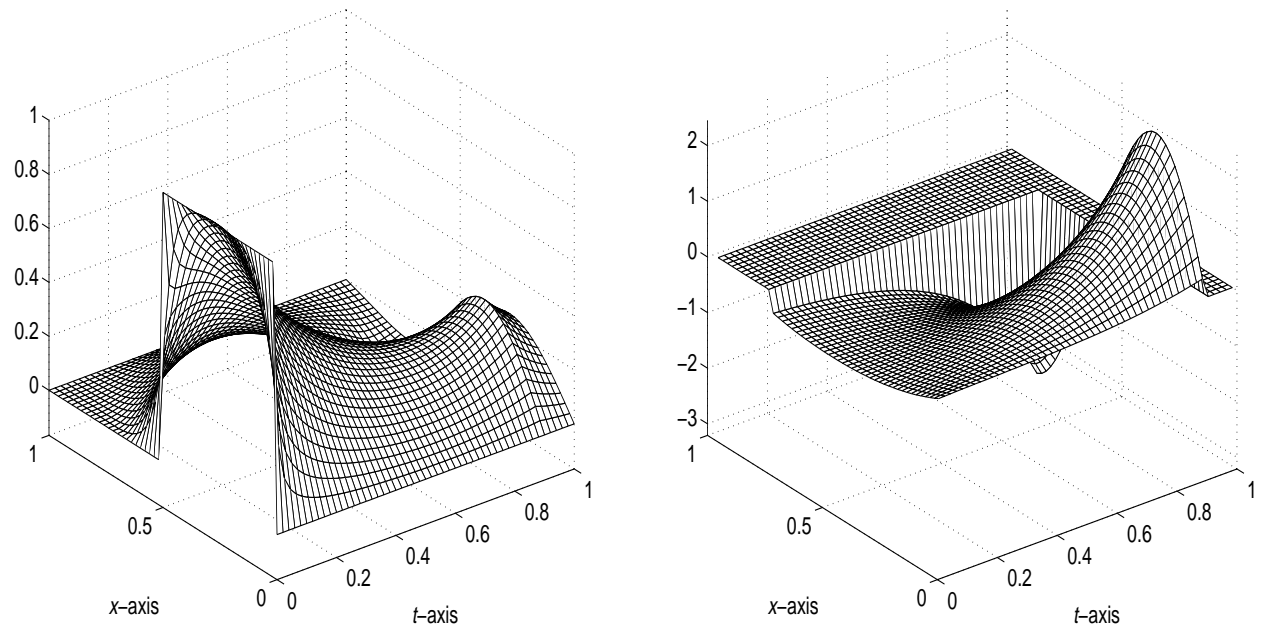

Figure 5. Optimal state and control for $N=M=50$.
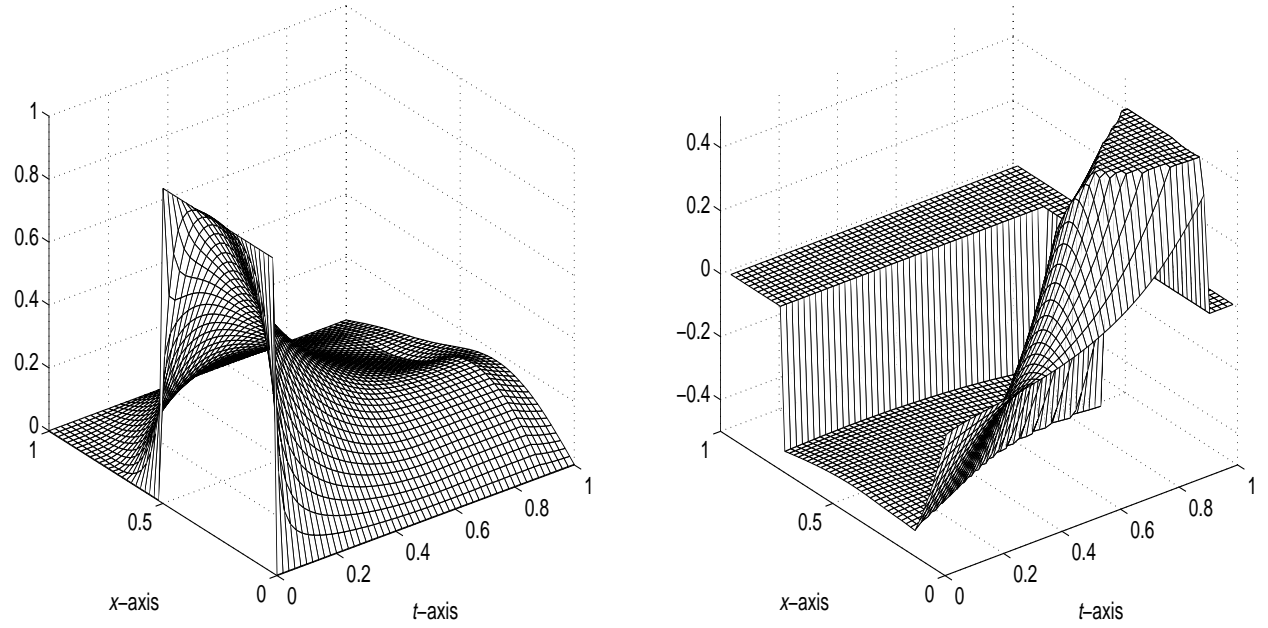

FiguRE 6. Optimal state and control for $u_{a}=-0.5=-u_{b}$ and $N=M=50$.

in Run 1. In Figure 5 the discrete solution is plotted. All three versions of the SQP method stop after four iterations. The needed CPU times are given in Table 7.

Now we take $u_{a}=-0.5=-u_{b}$. The discrete solution is presented in Figure 6 .

For $N=M=50$ the behavior of the SQP-method combined with Algorithm 1 is presented in Tables 8-10.

The values of the cost functional are presented in Table 11. 
TABLE 8. Number of inner iterations, residua, and CPU times for (SQP-LU).

\begin{tabular}{|c|c|c|c|}
\hline SQP iterations & Alg. 1 iterations & residuum & CPU time \\
\hline 1 & 7 & $1.25 \mathrm{e}-02$ & 86.7 seconds \\
\hline 2 & 5 & $1.12 \mathrm{e}-03$ & 148.0 seconds \\
\hline 3 & 3 & $6.54 \mathrm{e}-06$ & 96.8 seconds \\
\hline 4 & 3 & $5.04 \mathrm{e}-09$ & 96.9 seconds \\
\hline
\end{tabular}

TABLE 9. Number of inner iterations, residua, and CPU times for (SQP-GMRES).

\begin{tabular}{|c||c|r|r|}
\hline SQP iterations & Alg. 1 iterations & residuum & CPU time \\
\hline 1 & 7 & $1.25 \mathrm{e}-02$ & 59.6 seconds \\
\hline 2 & 5 & $1.12 \mathrm{e}-03$ & 67.6 seconds \\
\hline 3 & 3 & $6.54 \mathrm{e}-06$ & 32.4 seconds \\
\hline 4 & 3 & $5.04 \mathrm{e}-09$ & 30.7 seconds \\
\hline \multicolumn{2}{|l}{} & \multicolumn{2}{l}{$\sum=220.1$ seconds } \\
\cline { 3 - 4 }
\end{tabular}

TABLE 10. Number of inner iterations, residua, and CPU times for (SQP-GMRES-IN).

\begin{tabular}{|c||c|r|r|}
\hline SQP iterations & Alg. 1 iterations & residuum & CPU time \\
\hline 1 & 3 & $1.47 \mathrm{e}-02$ & 19.3 seconds \\
\hline 2 & 5 & $2.09 \mathrm{e}-03$ & 49.0 seconds \\
\hline 3 & 3 & $2.62 \mathrm{e}-05$ & 23.5 seconds \\
\hline 4 & 3 & $3.30 \mathrm{e}-08$ & 25.0 seconds \\
\hline \multicolumn{2}{|l}{} & & $\sum=146.7$ seconds \\
\cline { 4 - 5 }
\end{tabular}

TABLE 11. Values of the cost functional for $N=M=50$.

\begin{tabular}{|c||c|c|c|}
\hline & $u=0$ & $u \in L^{2}(Q)$ & $u \in U_{\text {ad }}$ \\
\hline$J\left(y^{*}, u^{*}\right)$ & 0.28873 & 0.22292 & 0.24455 \\
\hline
\end{tabular}

\section{Appendix A. Proof of Theorem 2.2}

To derive an estimate for $\|y(t)\|_{H}$ we choose $\varphi=y(t)$ in $(2.2 \mathrm{a})$. Then we get

$$
\frac{1}{2} \frac{\mathrm{d}}{\mathrm{d} t}\|y(t)\|_{H}^{2}+\nu\|y(t)\|_{V}^{2}=((f+b u)(t), y(t))_{H} .
$$

From (A.1), Poincaré's and Young's inequality we infer that

$$
\frac{\mathrm{d}}{\mathrm{d} t}\|y(t)\|_{H}^{2}+\nu\|y(t)\|_{H}^{2} \leq \frac{1}{\nu}\|(f+b u)(t)\|_{H}^{2} .
$$

Recall that in our case the Poincaré constant is equal to 1. Thus,

$$
\frac{\mathrm{d}}{\mathrm{d} t}\left(\mathrm{e}^{\nu t}\|y(t)\|_{H}^{2}\right) \leq \frac{\mathrm{e}^{\nu t}}{\nu}\|(f+b u)(t)\|_{H}^{2}
$$


Integrating (A.2) yields

$$
\|y(t)\|_{H}^{2} \leq \mathrm{e}^{-\nu t}\left\|y_{0}\right\|_{H}^{2}+\frac{\mathrm{e}^{\nu T}}{\nu}\|f+b u\|_{L^{2}(Q)}^{2} \leq\left\|y_{0}\right\|_{H}^{2}+\frac{2 \mathrm{e}^{\nu T}}{\nu}\left(\|f\|_{L^{2}(Q)}^{2}+\|b u\|_{L^{2}(Q)}^{2}\right) .
$$

Hence, there exists a constant $C_{1}>0$ depending on $\nu, f$, and $y_{0}$ such that

$$
\|y\|_{L^{\infty}(0, T ; H)} \leq C_{1}\left(1+\|b u\|_{L^{2}(Q)}\right) .
$$

Due to the differential equation we get

$$
\left\|y_{t}(t)\right\|_{V^{\prime}} \leq\left(\nu+\|y(t)\|_{H}\right)\|y(t)\|_{V}+\|(f+b u)(t)\|_{H} .
$$

This gives

$$
\left\|y_{t}\right\|_{L^{2}\left(0, T ; V^{\prime}\right)} \leq\left(\nu+\|y\|_{L^{\infty}(0, T ; H)}\right)\|y\|_{L^{2}(0, T ; V)}+\|f\|_{L^{2}(Q)}+\|b u\|_{L^{2}(Q)} .
$$

Integrating (A.1) over $(0, T)$ we end up with

$$
\|y\|_{L^{2}(0, T ; V)} \leq C_{2}\left(1+\|b u\|_{L^{2}(Q)}\right),
$$

where the constant $C_{2}>0$ depends on $\nu, y_{0}$, and $f$. From (A.4) and (A.5) we conclude that there exists a positive constant $C_{3}=C_{3}\left(\nu, y_{0}, f\right)$ such that

$$
\|y\|_{W(0, T)} \leq C_{3}\left(1+\|b u\|_{L^{2}(Q)}^{2}\right) .
$$

\section{Appendix B. Proof of Corollary 2.4}

We will prove that there exists a constant $C>0$ satisfying

$$
\|y\|_{\mathcal{W}(0, T)} \leq C\left(1+\|b u\|_{L^{2}(Q)}^{2}\right)
$$

for a constant $C>0$. Then the claim follows by an embedding argument. Choosing $\varphi=-y_{x x}(t)$ in (2.2a) we obtain

$$
\frac{1}{2} \frac{\mathrm{d}}{\mathrm{d} t}\|y(t)\|_{V}^{2}+\nu\left\|y_{x x}(t)\right\|_{H}^{2}=\left(\left(y y_{x}-f-b u\right)(t), y_{x x}(t)\right)_{H} .
$$

As in [13] (Th. 2) we find

$$
\|y(t)\|_{V} \leq\left\|y_{0}\right\|_{V}+C\left\|y_{0}\right\|_{H}^{3}+\|f+b u\|_{L^{2}(Q)}, \quad t \in[0, T] \text { a.e. }
$$

for a constant $C>0$. This bound gives together with (A.3) and (A.5)

$$
\|y\|_{L^{\infty}(0, T ; V)}+\|y\|_{L^{\infty}(0, T ; H)}+\|y\|_{L^{2}(0, T ; V)} \leq C\left(1+\|b u\|_{L^{2}(Q)}\right) .
$$

Integrating (B.7) we infer from Hölder's and Young's inequalities and (B.8) that

$$
\|y\|_{L^{2}\left(0, T ; H^{2}(\Omega) \cap V\right)} \leq C\left(1+\|b u\|_{L^{2}(Q)}\right) .
$$

Using the differential equation $y_{t}=\nu y_{x x}-y y_{x}+f+b u$, (B.8) and (B.9) we arrive at (B.6). 


\section{Appendix C. Proof of Proposition 2.7}

For $t \in[0, T]$ we introduce the bilinear form $a(t ; \cdot, \cdot)$ on $V \times V$ by

$$
a(t ; \varphi, \phi)=\int_{\Omega} \nu \varphi^{\prime} \phi^{\prime}+a_{1}(t) \varphi^{\prime} \phi+a_{2}(t) \varphi \phi \mathrm{d} x .
$$

Then, $a$ is continuous. In fact, we obtain

$$
|a(t ; \varphi, \phi)| \leq\left(\nu+\left\|a_{1}\right\|_{L^{\infty}(0, T ; H)}+\left\|a_{2}\right\|_{L^{\infty}(0, T ; H)}\right)\|\varphi\|_{V}\|\phi\|_{V}
$$

for $\varphi, \phi \in V$ and $t \in(0, T)$ a.e. Due to Agmon's inequality, see [16], there exists a constant $C_{A}>0$ satisfying

$$
\|\varphi\|_{L^{\infty}(\Omega)}^{2} \leq C_{A}\|\varphi\|_{H}\|\varphi\|_{V} \quad \text { for all } \varphi \in V
$$

Utilizing (C.10) and Young's inequality we find

$$
a(t ; \varphi, \varphi) \geq \frac{\nu}{2}\|\varphi\|_{V}^{2}-C\|\varphi\|_{H}^{2} \quad \text { for all } \varphi \in V \text { and } t \in[0, T] \text { a.e. }
$$

for a constant $C>0$ depending on $a_{1}, a_{2}$ and $\nu$. Hence, the existence of a unique solution $v \in W(0, T)$ of $(2.4)$ follows from [4]. Now we proceed by proving estimates. For that purpose we multiply the differential equation by $v$ and integrate over $\Omega$ and $(0, t)$ for $t \in(0, T)$. Using Hölder's inequality we get

$$
\begin{aligned}
\frac{1}{2}\|v(t)\|_{H}^{2}+\nu\|v\|_{L^{2}(0, t ; V)}^{2} \leq & \frac{1}{2}\left\|v_{0}\right\|_{H}^{2}+\|g\|_{L^{2}(Q)}\|v\|_{L^{2}(0, t ; H)}+\left\|a_{1}\right\|_{C([0, T] ; H)}\left\|v_{x}\right\|_{L^{2}(0, t ; H)}\|v\|_{L^{2}\left(0, t ; L^{\infty}(\Omega)\right)} \\
& +\left\|a_{2}\right\|_{C([0, T] ; H)}\|v\|_{L^{2}\left(0, t ; L^{\infty}(\Omega)\right)}\|v\|_{L^{2}(0, t ; H)} .
\end{aligned}
$$

From Agmon's and Young's inequalities it follows that

$$
\|v(t)\|_{H}^{2}+\nu\|v\|_{L^{2}(0, t ; V)}^{2} \leq C\left(\|g\|_{L^{2}(Q)}^{2}+\left\|v_{0}\right\|_{H}^{2}+\|v\|_{L^{2}(0, t ; H)}^{2}\right)
$$

for $t \in[0, T]$. Due to Gronwall's inequality we find

$$
\|v(t)\|_{H}^{2} \leq C\left(\|g\|_{L^{2}(Q)}^{2}+\left\|v_{0}\right\|_{H}^{2}\right) \quad \text { for } t \in[0, T]
$$

Thus,

$$
\|v\|_{L^{\infty}(0, T ; H)} \leq C\left(\|g\|_{L^{2}(Q)}+\left\|v_{0}\right\|_{H}\right)
$$

Using (C.12) we infer from (C.11)

$$
\|v\|_{L^{2}(0, T ; V)} \leq C\left(\|g\|_{L^{2}(Q)}+\left\|v_{0}\right\|_{H}\right) .
$$

Now the estimate in the $W(0, T)$-norm follows from (C.12, C.13) and $v_{t}=\nu v_{x x}-a_{1} v-a_{2} v_{x}+g$. We continue by proving the second estimate. Therefore, we multiply the differential equation with $-v_{x x}$ and integrate over $\Omega$ and $(0, t)$ for $t \in(0, T)$. Hölder's inequality yields

$$
\begin{aligned}
\frac{1}{2}\|v(t)\|_{V}^{2}+\nu\left\|v_{x x}\right\|_{L^{2}(0, t ; H)}^{2} \leq & \frac{1}{2}\left\|v_{0}\right\|_{V}^{2}+\|g\|_{L^{2}(Q)}\left\|v_{x x}\right\|_{L^{2}(0, t ; H)}+\left\|a_{1}\right\|_{C([0, T] ; H)}\left\|v_{x}\right\|_{L^{2}\left(0, t ; L^{\infty}(\Omega)\right)}\left\|v_{x x}\right\|_{L^{2}(0, t ; H)} \\
& +\left\|a_{2}\right\|_{C([0, T] ; H)}\|v\|_{L^{2}\left(0, t ; L^{\infty}(\Omega)\right)}\left\|v_{x x}\right\|_{L^{2}(0, t ; H)}
\end{aligned}
$$


for $t \in[0, T]$ a.e. Using Agmon's and Young's inequalities, equations (C.12, C.13) and the estimate $\left\|v_{0}\right\|_{H} \leq$ $\left\|v_{0}\right\|_{V}$ we conclude

$$
\|v(t)\|_{V}^{2}+\nu\left\|v_{x x}\right\|_{L^{2}(0, t ; H)}^{2} \leq C\left(\|g\|_{L^{2}(Q)}^{2}+\left\|v_{0}\right\|_{V}^{2}\right) \quad \text { for } t \in[0, T]
$$

Hence,

$$
\|v\|_{L^{\infty}(0, T ; V)}+\left\|v_{x x}\right\|_{L^{2}(0, T ; H)} \leq C\left(\|g\|_{L^{2}(Q)}+\left\|v_{0}\right\|_{V}\right) .
$$

From (C.12-C.14) and $v_{t}=\nu v_{x x}-a_{1} v-a_{2} v_{x}+g$ the stated estimate follows.

The authors would like to thank Dr. M. Hintermüller for helpful discussions about the primal-dual active set strategy.

\section{REFERENCES}

[1] R.A. Adams, Sobolev Spaces. Academic Press, New York (1975).

[2] W. Alt, The Lagrange-Newton method for infinite-dimensional optimization problems. Numer. Funct. Anal. Optim. 11 (1990) 201-224.

[3] M. Bergounioux, K. Ito and K. Kunisch, Primal-dual strategy for constrained optimal control problems. SIAM J. Control Optim. 35 (1997) 1524-1543.

[4] R. Dautray and J.-L. Lions, Mathematical Analysis and Numerical Methods for Science and Technology, Vol. 5: Evolution Problems I. Springer-Verlag, Berlin (1992).

[5] A.L. Dontchev, Local analysis of a Newton-type method based on partial linearization, in Proc. of the AMS-SIAM Summer Seminar in Applied Mathematics on Mathematics and Numerical Analysis: Real Number Algorithms, edited by J. Renegar, M. Shub and S. Smale. AMS, Lectures in Appl. Math. 32 (1996) 295-306.

[6] A.L. Dontchev, W.W. Hager, A.B. Poore and B. Yang, Optimality, stability, and convergence in optimal control. Appl. Math. Optim. 31 (1995) 297-326.

[7] H. Goldberg and F. Tröltzsch, On the Lagrange-Newton-SQP method for the optimal control of semilinear parabolic equations. Optim. Methods Softw. 8 (1998) 225-247.

[8] M. Heinkenschloss and F. Tröltzsch, Analysis of the Lagrange-SQP-Newton Method for the Control of a Phase-Field Equation. Control Cybernet. 28 (1999) 177-211.

[9] M. Hintermüller, A primal-dual active set algorithm for bilaterally control constrained optimal control problems. Spezialforschungsbereich F 003, Optimierung und Kontrolle, Projektbereich Optimierung und Kontrolle, Bericht No. 146 (submitted).

[10] M. Hinze and K. Kunisch, Second order methods for time-dependent fluid flow. Spezialforschungsbereich F 003, Optimierung und Kontrolle, Projektbereich Optimierung und Kontrolle, Bericht No. 165 (submitted).

[11] K. Ito and K. Kunisch, Augmented Lagrangian-SQP-Methods for nonlinear optimal control problems of tracking type. SIAM J. Control Optim. 34 (1996) 874-891.

[12] K. Kunisch and A. Rösch, Primal-dual strategy for parabolic optimal control problems. Spezialforschungsbereich F 003, Optimierung und Kontrolle, Projektbereich Optimierung und Kontrolle, Bericht No. 154 (submitted).

[13] H.V. Ly, K.D. Mease and E.S. Titi, Some remarks on distributed and boundary control of the viscous Burgers equation. Numer. Funct. Anal. Optim. 18 (1997) 143-188.

[14] S.M. Robinson, Strongly regular generalized equations. Math. Oper. Res. 5 (1980) 43-62.

[15] R. Temam, Navier-Stokes Equations. North-Holland, Amsterdam, Stud. Math. Appl. (1979).

[16] R. Temam, Infinite-Dimensional Dynamical Systems in Mechanics and Physics. Springer-Verlag, New York, Appl. Math. Sci. 68 (1988).

[17] F. Tröltzsch, Lipschitz stability of solutions to linear-quadratic parabolic control problems with respect to perturbations. Dynam. Contin. Discrete Impuls. Systems 7 (2000) 289-306.

[18] F. Tröltzsch, On the Lagrange-Newton-SQP method for the optimal control of semilinear parabolic equations. SIAM J. Control Optim. 38 (1999) 294-312.

[19] S. Volkwein, Mesh-Independence of an Augmented Lagrangian-SQP Method in Hilbert Spaces and Control Problems for the Burgers Equation, Ph.D. Thesis. Department of Mathematics, Technical University of Berlin (1997).

[20] S. Volkwein, Augmented Lagrangian-SQP techniques and optimal control problems for the stationary Burgers equation. Comput. Optim. Appl. 16 (2000) 57-81.

[21] S. Volkwein, Distributed control problems for the Burgers equation. Comput. Optim. Appl. 18 (2001) 133-158.

[22] S. Volkwein, Optimal control of a phase-field model using the proper orthogonal decomposition. Z. Angew. Math. Mech. 81 (2001) 83-97. 